\title{
Machine-learning-based children's pathological gait classification with low-cost gait-recognition system
}

Linghui Xu ( $\nabla$ linghui10@foxmail.com )

Zhejiang University https://orcid.org/0000-0001-8489-2920

Jiansong Chen

Zhejiang University School of Medicine

Fei Wang

China Academy of Art

Yuting Chen

Yanshan University

Wei Yang

Zhejiang University

Canjun Yang

Zhejiang University

\section{Research Article}

Keywords: pathological gait recognition, pressure-sensor array, gait classification, feature extraction

Posted Date: March 24th, 2021

DOI: https://doi.org/10.21203/rs.3.rs-327945/v1

License: (c) (i) This work is licensed under a Creative Commons Attribution 4.0 International License.

Read Full License 


\section{Machine-learning-based children's pathological gait classification}

\section{with low-cost gait-recognition system}

Linghui $\mathrm{Xu}^{1,3}$, Jiansong $\mathrm{Chen}^{2}$, Fei Wang ${ }^{4}$, Yuting Chen ${ }^{5}$, Wei Yang ${ }^{1,3}$, Canjun Yang ${ }^{1,3}$

${ }^{1}$ Ningbo Research Institute, Zhejiang University, Ningbo 315100, China

${ }^{2}$ Department of Orthopedics, The Children's Hospital, Zhejiang University School of Medicine, Hangzhou 310006, China

${ }^{3}$ State Key Laboratory of Fluid Power and Mechatronic Systems, Zhejiang University, Hangzhou 310027, China

${ }^{4}$ Industrial Design Department of the Art and Design Institute, China Academy of Art, Hangzhou 310024, China

${ }^{5}$ Hebei Heavy Machinery Fluid Power Transmission and Control Lab, Yanshan University, Qinhuangdao 066004, China

Corresponding author: Wei Yang (e-mail: simpleway@zju.edu.cn) 
Abstract

Background: Pathological gaits of children may lead to terrible diseases, such as osteoarthritis or scoliosis. By monitoring the gait pattern of a child, proper therapeutic measures can be recommended to avoid the terrible consequence. However, low-cost systems for pathological gait recognition of children automatically have not been on market yet. Our goal was to design a low-cost gaitrecognition system for children with only pressure information.

Methods: In this study, we design a pathological gait-recognition system (PGRS) with an $8 \times 8$ pressure-sensor array. An intelligent gait-recognition method (IGRM) based on machine learning and pure plantar pressure information is also proposed in static and dynamic sections to realize high accuracy and good real-time performance. To verifying the recognition effect, a total of seventeen children were recruited in the experiments wearing PGRS to recognize three pathological gaits (toe in, toe out, and flat) and normal gait. Children are asked to walk naturally on level ground in the dynamic section or stand naturally and comfortably in the static section. The evaluation of the performance of recognition results included stratified 10 -fold cross-validation with recall, precision, and a time cost as metrics.

Results: The experimental results show that all of the IGRMs have been identified with a practically applicable degree of average accuracy either in the dynamic or static section. Experimental results indicate that the IGRM has $92.41 \%$ and $97.79 \%$ recognition accuracy respectively in the static and dynamic sections. And we find methods in the static section have less recognition accuracy due to the unnatural gesture of children when standing.

Conclusions: In this study, a low-cost PGRS has been verified and realize feasibility, highly average precision, and good real-time performance of gait recognition. The experimental results reveal the potential for the computer supervision of non-pathological and pathological gaits in the plantarpressure patterns of children and for providing feedback in the application of gait-abnormality rectification.

Keywords: pathological gait recognition, pressure-sensor array, gait classification, feature extraction 


\section{Background}

Children are prone to have pathological gaits when starting toddling, which may cause osteoarthritis, scoliosis, or other debilitating diseases. To monitoring the pathological gait pattern of a human, various bio-signals are adopted among which kinematics information and plantar-pressure show more potential for their easy to measure and explain [1,2]. These high-dimensional bio-signals indicate complex states of human muscles and joints [3], which cause difficulty to interpret directly by conventional kinematics or kinetics. However, machine learning (ML) recently has more potential to deal with the large-data-driven pattern-recognition problems with the development of computer technology $[1,4]$.

Employing computer-vision technology, kinematics information can be easily acquired. Elham et $a l$. acquired gait features including angles, velocity, and acceleration of the joints based on Kinect skeletal tracking sequences [5]. Two ML approaches, an instance-based discriminative classifier and a dynamical generative classifier, were examined to distinguish between healthy and pathological gaits. F1-score of the former can reach up to $96 \%$ when walking at a fast pace. Javier et al. developed vision-based gait-impairment analysis for aided diagnosis [6]. A number of semantic and normalized gait features were computed from a single video to provide samples under eight different walking styles: one normal and seven impaired patterns. Several statistical studies were carried out to prove the sensitivity of features in measuring the expected pathologies. Zakaria et al. [7] classified Autism Spectrum Disorder (ASD) children's gait from normal gait. Gait features were the absolute or relative Cartesian coordinates of 20 joints of the subject measured by a depth camera. Various methods were used to classify these two gaits, among which support vector machine (SVM) classifier has the lowest accuracy, 98.67\%, and Naives Bayes classifier has the highest accuracy, 99.66\%. Chen et al. [8] proposed a gait classification and develop a simple and efficient method for the quantification method for parkinsonian gait from monocular video imaging based on kernel-based principal component analysis.

Plantar pressure contains abundant gait-pattern information, which can be used to reasonably predict and explain human physiological diseases [2]. However, it has high dimensionality, temporal 
dependence, high variability, correlations between curves, and nonlinear relationship features [4]. Mei et al. $[9,10]$ analyzed the force of center of pressure $(\mathrm{CoP})$ sample entropy characteristics among the four types, pes cavus, pes valgus, hallux valgus, and normal feet, using the Footscan system. The study showed that dynamic characteristics of $\mathrm{CoP}$ progression contain information of the foot type. Zhu et al. [11] developed an umbilical data-acquisition system to measure the pressure between the foot and shoe during walking that had seven force-sensitive resistors (FSRs) on the surface of each insole of a pair of extra-depth shoes. The team found that a shuffling gait with short steps would increase the period of foot flat and thus minimize any excessive local plantar pressures [12]. Lin et al. [13] employed dynamic features derived from tracking gait to recognize individuals. The self-organizingmap (SOM) neural network (NN) algorithm and SVM were used in both schemes for data classification. Experiments showed that a higher recognition rate was achieved with the method using all of the plantar pressure sensor-cell values during walking regardless of the algorithm used, which suggested that the foot-pressure distribution of gait is a suitable feature for gait recognition. Sazonov et al. [14] built a $\mathrm{NN}$ to predict geriatric patterns using plantar pressure and heel acceleration information, whose classification accuracy was $91.6 \%$ on average. Data for training the NN were collected by sensor shoes with 34 pressure-sensing elements uniformly distributed across the foot and a $2 \mathrm{D}$ accelerometer. Based on a shoe-integrated system with an inertial measurement unit (IMU), four FSRs, and a bend sensor, Chen et al. [15] applied principal component analysis (PCA) and SVM for multi-pattern classification (toe in, toe out, over-supination, heel walking, and normal pattern). A total of four subjects tested the shoe-integrated device in outdoor environments. Experimental results of the four subjects demonstrated that the proposed method was robust and highly accurate up to $90 \%$. More interestingly, the study showed that insole sensors played a more important role in solving classification problems than IMUs.

Here, three foot types of children including toe in, toe out, and flat feet are concerned. According to big database research, toe in, toe out, and flat feet are the most common reasons for parental concerns and referral for a specialist opinion about their children's gait [16]. The vast majority of pathological gait may correct spontaneously if left untreated [17-21] due to the underdeveloped skeleton of children, which makes it more of a concern to the parents than to the podiatrist. However, 
since the theoretical explanation and analysis for the self-healing capability is unclear, the child's gait still needs constant attention. Thus, designing a device for parents to watch children's gait periodically at home is of high necessity. Many underlying causes are lying in the hip joint, femur, or tibia or the hindfoot or forefoot may lead to in- or out- toeing gait [16]. The most straightforward physical examination method is measuring foot progression angle (FPA) which describes the orientation of the child's foot to the direction of progression. For a normal child, FPA is slightly out-toed $\left(+10^{\circ}\right)$ with a range from $-3^{\circ}$ to $+20^{\circ}[22,23]$. If FPA is less than $-3^{\circ}$, a child is considered to have an in-toeing gait and if it is more than $20^{\circ}$, a child is considered to have an out-toeing gait. And FPA can influence the distribution of the plantar pressure. Rosenbaum (2013) [24] found that in-toeing increasingly loads the lateral aspects of the midfoot and forefoot by as much as $61 \%$ and $49 \%$, respectively, whereas outtoeing intensifies the load on the medial aspect, i.e. predominantly the medial midfoot and medial forefoot by as much as $72 \%$ and $52 \%$. Traditionally, a flat foot can also be diagnosed by measuring the area of contact between the foot and the ground $[20,25]$. As far as we are concerned, there is no related low-cost system for pathological gait recognition of children automatically for ordinary parents with only pressure information. The contributions of this study are the following.

1) A low-cost pathological gait-recognition system (PGRS) with an $8 \times 8$ pressure sensor array is built. With this system, parents can watch children's gait state daily without the need to consult a podiatrist frequently in hospital. This not only reduces the worry and anxiety of parents but also improves the efficiency of podiatrists, reducing the pointless counseling for childhood gait.

2) A highly accurate and fast intelligent gait-recognition method (IGRM) is realized in static and dynamic situations utilizing only plantar-pressure data.

3) The effects of the algorithm with plantar pressure data collected in both static and dynamic sections are compared. And the research shows that the IGRMs in the dynamic section have higher average accuracy than those in the static section.

\section{Results}

In this part, a low-cost PGRS is built to measure children's plantar-pressure data during walking and a series of experiments are designed to verify the availability of the IGRM. Several typical gaits, 
namely, toe in, toe out, flat, and normal, are involved. All of the subjects signed informed consent forms before experiments.

\section{Pathological Gait-Recognition System}

As shown in Fig. 1, a PGRS consists of an $8 \times 8$ pressure-sensor array, a signal-collecting circuit, a micro-computing unit, and a wearable shoe-integrated mechanism is designed.

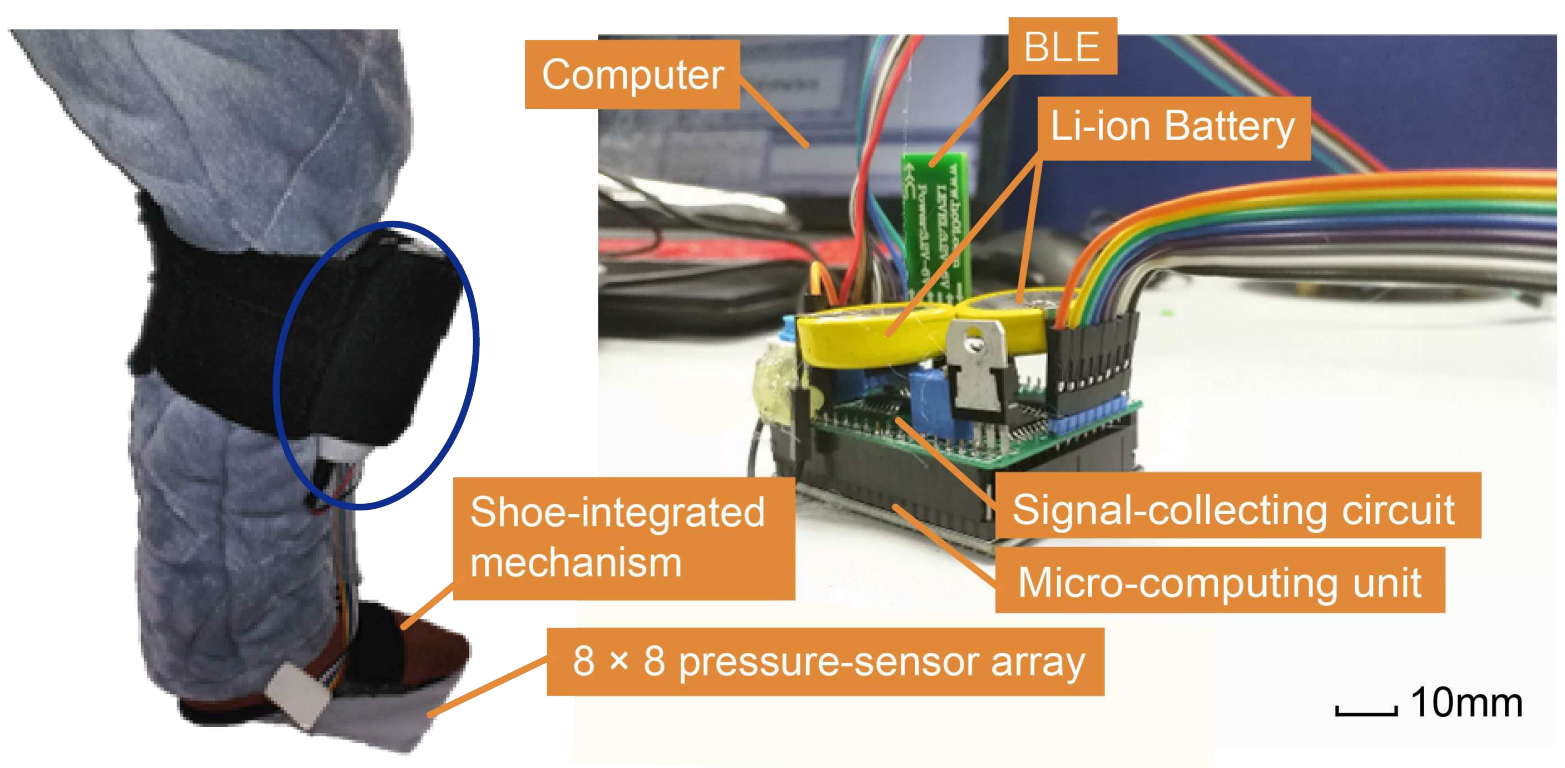

Fig. 1 Pathological gait-recognition system. $8 \times 8$ sensor array is under the slipper and controller attached to the child's leg with hook-and-loop fasteners. The control circuit board contains a signal-collecting circuit, a lowenergy Bluetooth device (HC-42 with Bluetooth 5.0, HuiCheng Information Technology Co., Ltd., China), STM32F103 controller, and two 4.2-V Li-ion batteries. The signal-collecting circuit operates with $5 \mathrm{~V}$ of power generated by an LM7805 unit (KIA7805AP, three-terminal positive voltage regulator of 5 V, KEC, China) and the STM32F103 circuitry operates with $3.3 \mathrm{~V}$ generated by an AMS117 unit (low-dropout-voltage regulator with fixed 3.3 V, Advanced Monolithic Systems, Inc.)

The plantar pressure of adults is in the range of $0-1000 \mathrm{kPa}$ during walking [26], and the maximal pressure can up to approximately $1400 \mathrm{kPa}$ when doing sports. Based on weight conversion, children's plantar pressure is estimated to be in the range of 0 to $700 \mathrm{kPa}$. Therefore, the pressuredetection range of the sensor array is chosen as $0-1000 \mathrm{kPa}$. The $8 \times 8$ piezoresistive sensor array structure and parameter characteristics of this series (Changzhou Roxi Electronic Technology Co. 
LTD, China) are shown in Fig. 2a and b. The relationship of load pressure $P$ onto the sensor and its resistance $R_{x}$ can be described as:

$$
R_{x}=\frac{1}{P} K_{p-r}
$$

where $K_{p-r}$ is the sensor characteristic constant. All the test results of the $8 \times 8$ sensors' performance using the control circuit mentioned below are shown in Fig. 2c, indicating its good repeatability and linearity.
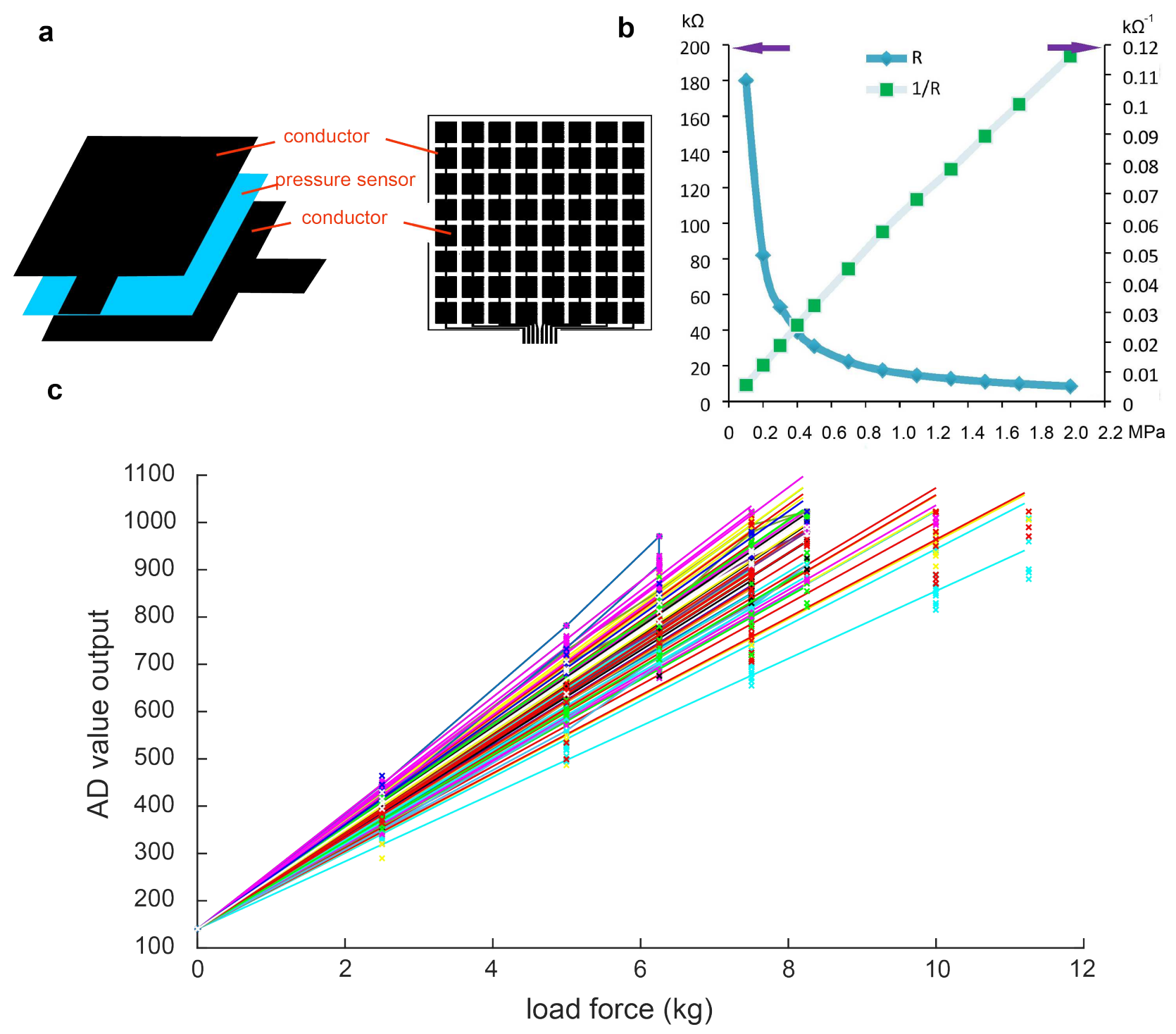

Fig. 2 a The three-layer structure of the piezoresistive sensor. b Piezoresistive sensor pressure-resistor characteristic curve. c Sensor calibration

For the signal-collecting circuit and the micro-computing unit, as the schematic is shown in Fig. 3 a, a microcontroller (STM32F103C8T6 with Cortex-M3 core and a maximum CPU speed of $72 \mathrm{MHz}$; 
STMicroelectronics Corp., USA) was chosen as its control core. Noninverting amplifier converts resistor of the sensor $R_{x}$ to voltage $V_{\text {out }}$. The charging Resistor $R_{0}$ used to stable the ADC output is 1 $\mathrm{K} \Omega$ [27]. We use two pieces of quad bilateral switch HCF4066B chip (STMicroelectronics Corp., USA) to compose one 8:1 analog switch. The state of the switch is controlled by the logic level of $\mathrm{I} / \mathrm{O}$ from the microcontroller. The microcontroller is programmed to select the sensor block one by one using two 8:1 analog switches and to read the sensor block's output. Two 4.2-V Li-ion batteries provide power, generating 5V with LM7805 (KIA7805AP, three-terminal positive voltage regulator of $5 \mathrm{~V}, \mathrm{KEC}$, China) for the signal-collecting circuit and 3.3V with AMS117 (low-dropout-voltage regulator with fixed 3.3 V, Advanced Monolithic Systems, Inc.) for the microcontroller.

Through an amplifier and feedback resistance $R_{f}$, the output voltage $V_{\text {out }}$ of the amplifier is linear to the pressure sensor received. Defining $D_{\text {output }}, V_{\text {adc_ref }}$ and $m$ as the digital value received by the analog-to-digital converter (ADC), the reference voltage and resolution of the ADC, respectively, $V_{\text {out }}$ is related to the resistance of a specific sensor. We have

$$
\begin{gathered}
V_{\text {out }}=\frac{R_{x}+R_{f}}{R_{x}} V_{\text {ref }}, \\
D_{\text {output }}=2^{m} \frac{V_{\text {out }}}{V_{\text {adc_ref }}}=2^{m}\left(\frac{k_{P-R}+P \times R_{f}}{k_{P-R}}\right) \frac{V_{\text {ref }}}{V_{\text {adc_ref }}},
\end{gathered}
$$

where $V_{\text {adc_ref }}$ is $5 \mathrm{~V}, V_{\text {ref }}$ is $0.5 \mathrm{~V}$ and $m$ is 10 in the experiments. We can see that $D_{\text {output }}$ is relevant to load pressure $P$ only.

During the test, it was found that the pressure on one sensor would lead to an unexpected output of the rest of the sensors. Coupling is found between sensor blocks caused by current path 2 and path 3 when measuring the sensor value at $(1,1)$, where (No.1, No.2) means the sensor block at row channel No.1 and column channel No.2, as shown in Fig. 3a. The closer the distance between two sensors is, the stronger their interactions are. To extract individual sensor resistor value one after another from the $8 \times 8$ sensor array and eliminate the coupling between each sensor, as shown in Fig. $3 b[28,29]$, the active sensor during readout was selected by grounding one of the 8:1 analog switch channels on the 
column, while other channels are pulling up to $V_{\text {ref }}$. The same control operation is applied to row pins of the $8 \times 8$ sensor array at the same time. So that the current path except path 1 such as path 2 will be cut off. The sampling result showing in Fig. $3 \mathrm{c}$ indicates that the ADC output value (maximum is 1024) of a certain sensor influenced by other sensors is within $10(32.2 \mathrm{mV}$ output, corresponding to $0.282 \mathrm{~kg}$ loaded), which is much better than the previous maximum, almost $70(225 \mathrm{mV}$ output, corresponding to $1.977 \mathrm{~kg}$ loaded).

a
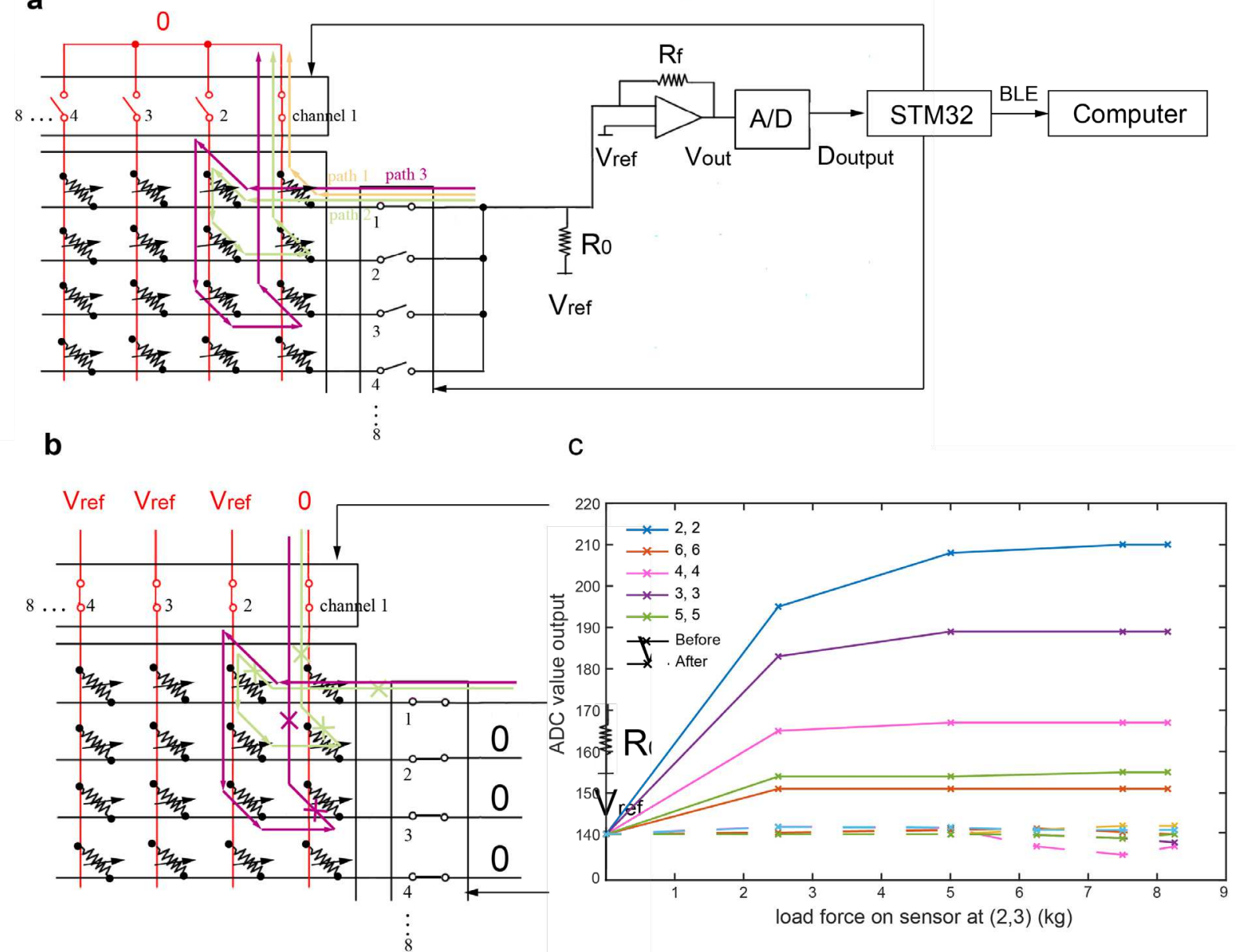

Fig. 3 Piezoresistive sensor array scanner electronic schematic. a Schematic before pressure decoupling. A microcontroller is used to select the sampling row and column channel by controlling quad bilateral switch CD4066 chip, while other unselected channels are remaining high resistance. Coupling output is found between sensor blocks as path 1 and path 2 show. b Schematic after pressure decoupling. When a certain row and column channels are selected (row channel 1 and column channel 1 is selected here), we pull down other row channels to ground and pull up other column channels to Vref, such that path 2 and path 3 will be cut off and path 1 will remain. Coupling output between sensor blocks can be eliminated. $\mathbf{c}$ The load force on the sensor block at $(2,3)$ 
has less influence on other sensors' value output after sensor array decoupling. The dotted line represents data after sensor array decoupling and the solid line represents data before sensor array decoupling

\section{Gait-Pressure-Data Collection}

A GUI-based program using MatLab R2016a (MathWorks, USA) was designed to make the pressure information more available and more visual. Its flow chart is shown in Fig. 4, which has functions of filtering the pressure data, showing the real-time pressure data curve of a specific plantarpressure sensor, saving pressure data in the computer, and obtaining the gait-pattern results.

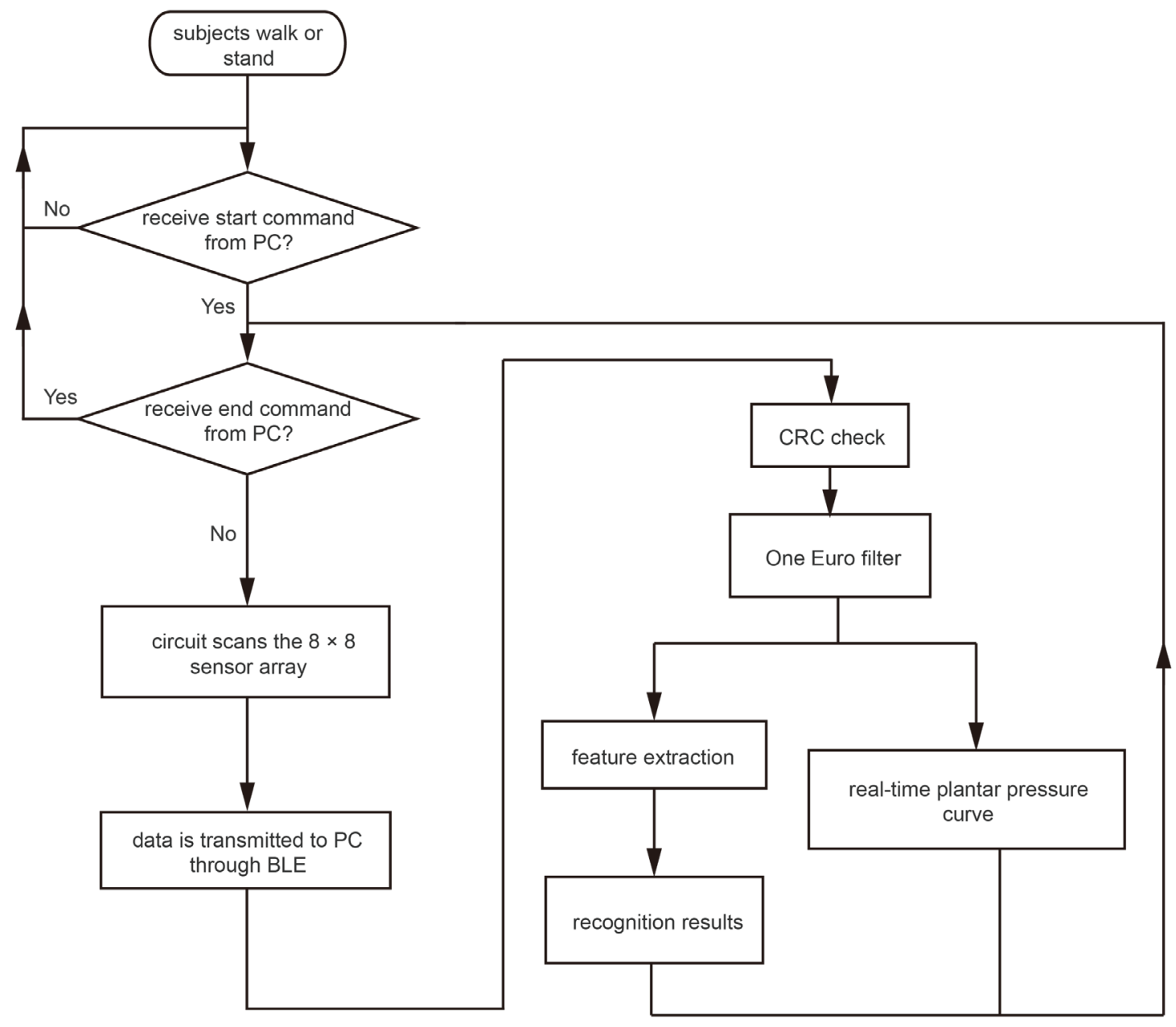

Fig. 4 GUI background flowchart. GUI/PC can receive commands from people in the GUI and use BLE to communicate with the controller system

To meet the expected situation where people normally want to have low disturbance in slow signals and low lag in fast signals, the filter used in the aforementioned program is One Euro filter [30]. And [29] has found that a straightforward linear relationship between cutoff frequency and the 
absolute speed of the input signal works well. To construct One Euro filter, the frequency spectrum information of input is studied. Based on the experiment data collected at the Children's Hospital, Zhejiang University School of Medicine, the frequency spectrum of plantar-pressure data of the children shows almost all information is located in $0-10 \mathrm{~Hz}$, which is similar to the features found by Hangqi Wei [31] about adult plantar pressure.

As shown in Table 1, seventeen subjects who had undergone gait diagnosis by a specialist were recruited to participate in the experiment. The experiments are divided into two sections, dynamic and static section. The experimental procedures of the dynamic section are shown in Fig. 5. Subjects are asked to walk naturally for 10 meters two times with a two-minute gap on level ground. In the static section, children are expected to stand naturally and comfortably for about ten seconds five times.

Table 1 Experimental subjects' basic information.

\begin{tabular}{cccc}
\hline Index & Age (years) & Diagnosis & Male/Female \\
\hline 1 & 6 & Flat & Female \\
2 & 10 & Flat & Female \\
3 & 8 & Flat & Male \\
4 & 9 & Flat & Male \\
5 & 10 & Flat & Female \\
6 & 7 & Flat & Female \\
7 & 9 & Flat & Male \\
8 & 9 & Flat & Male \\
9 & 10 & Flat & Male \\
10 & 8 & Normal & Female \\
11 & 5 & Normal & Female \\
12 & 6 & Normal & Male \\
13 & 6 & Normal & Female \\
14 & 8 & Toe in & Female \\
15 & 9 & Toe in & Male \\
16 & 8 & Toe out & Female \\
17 & 9 & Toe out & Male \\
\hline
\end{tabular}




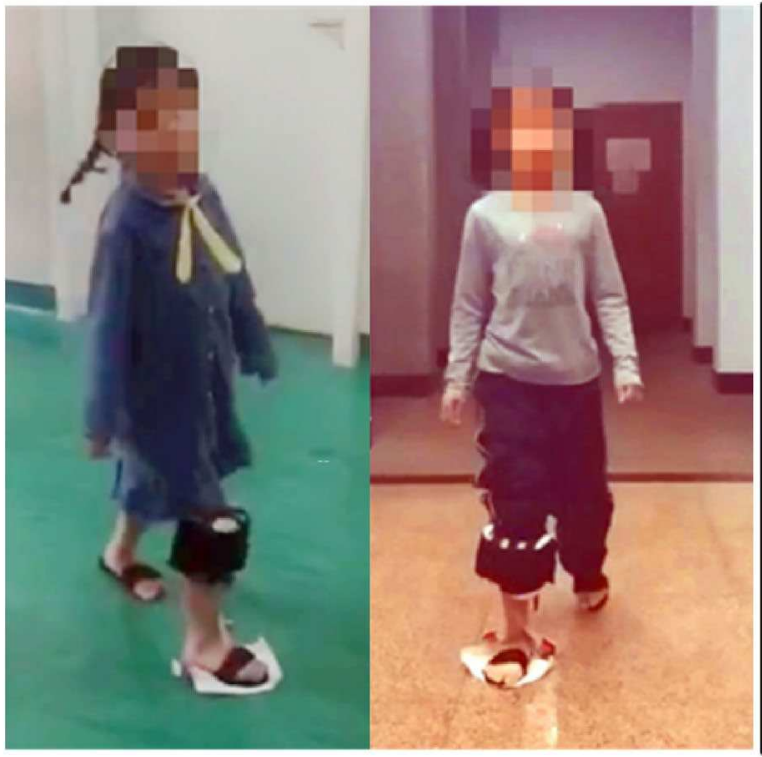

a

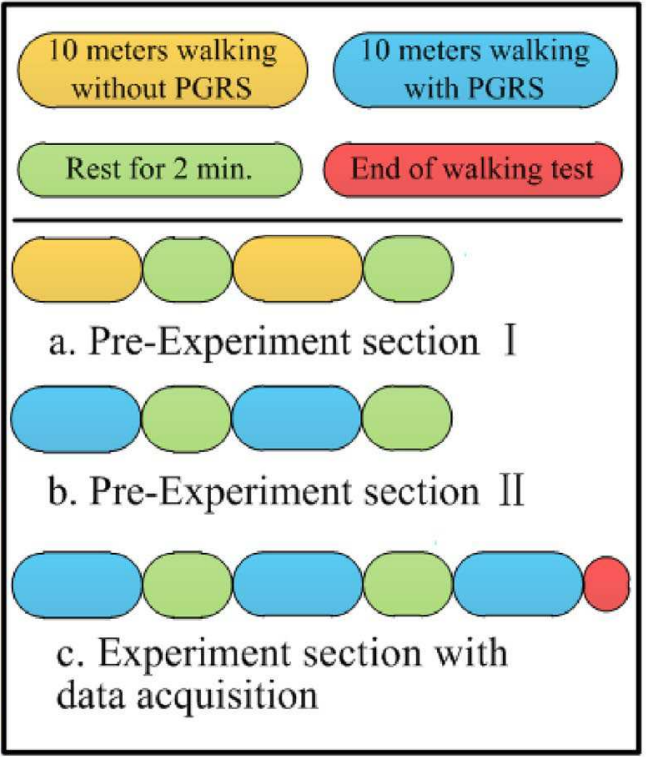

b

Fig. 5 Experiment in the dynamic section. a snapshots of subjects. b Experimental procedure of the dynamic section

One example of the collected plantar-pressure data in the dynamic section is shown in Fig. 6a. The plantar-pressure curve can be divided into several gait phases: initial contact (IC), loading response (LR), mid-stance (MS), terminal stance (TS), pre-swing (PS), and swing phase (SP). And typical toe in, toe out, and normal foot-pressure distribution in static-section is shown in Fig. 6 b.

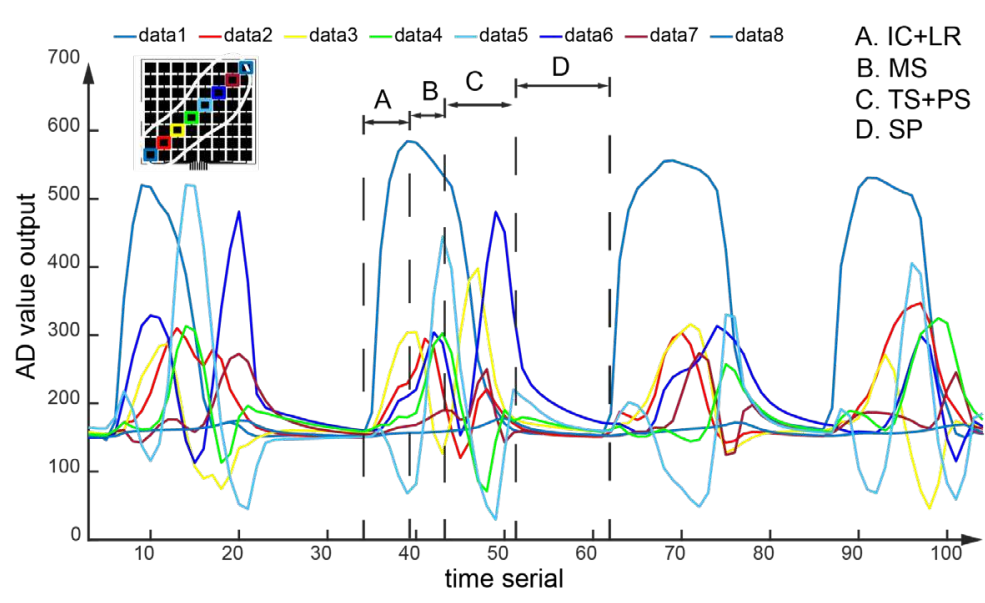

a

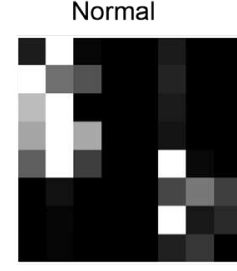

Toe out

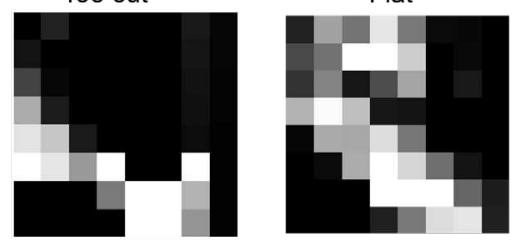

b

Fig. 6 Plantar-pressure data acquisition results. a Curve of plantar pressure with time during level-ground walking in the dynamic-section experiment. b Typical toe in, toe out, and normal foot-pressure distribution in the static-section experiment. The plantar pressure value is expressed in different grey scales. Pure black means zero pressure, pure white means the largest pressure 


\section{Gait-Recognition Results}

The IGRM is coded in Python with scikit-learn library. PCA or linear discriminant analysis (LDA) is used to reduce plantar-pressure data dimension in both dynamic and static sections. Coefficient gamma of RBF kernel in SVM is chosen as $1 /($ features $\times$ variance of data) and penalty parameter $\mathrm{C}$ is 1. The layer of the $\mathrm{NN}$ is 3 and the neuron number of the hidden layer is 10 . The total experiment samples are 20000 in which normal, toe in, toe out, and flat have 5000 samples respectively in the dynamic section and 1308 in which normal, toe in, toe out, and flat have 327 samples respectively in the static section. The scheme of evaluation included stratified 10 -fold cross-validation with recall, precision, and a time cost as metrics. All statistical analysis was performed using SPSS version 22 (SPSS Inc., Chicago, IL, USA). the time cost is calculated by predicting 2000 samples in the dynamic section or 130 samples in the static section using the same computer.

Experimental performance of dynamic and static sections respectively are shown in Table 2 and Table 3. Fig. 7 shows the distribution of the accuracy of different types of algorithms which are evaluated by 10 -fold cross-validation and the average time cost per fold. The classification result of classification models to all the samples is shown in the average confusion matrix in Fig. 8.

Table 2 Experimental results data of dynamic section.

\begin{tabular}{ccccc}
\hline Algorithm & Recall & $\begin{array}{c}\text { Average precision } \\
(\%)\end{array}$ & $\begin{array}{c}\text { Time cost } \\
(\mathrm{ms})\end{array}$ & $\begin{array}{c}\text { Accuracy/time cost } \\
\text { P-value }\end{array}$ \\
\hline LDA+SVMrbf & $0.96 / 1.00 / 0.96 / 0.93$ & 96.24 & 6.10 & $0.991 /<<0.01$ \\
LDA+SVMlin & $1.00 / 1.00 / 1.00 / 1.00$ & 97.79 & 4.40 & -- \\
& & 87.24 & 75.20 & $0.007 /<<0.01$ \\
PCA+SVMlin & $0.68 / 1.00 / 0.99 / 0.81$ & 94.13 & 27.40 & $0.107 /<<0.01$ \\
PCA+SVMrbf & $0.90 / 1.00 / 1.00 / 0.86$ & 97.38 & 5.10 & $0.702 / 0.064$ \\
LDA+NN & $0.96 / 1.00 / 0.97 / 0.92$ & & &
\end{tabular}

"---" means the P-value of this column is computed based on this algorithm. In the Recall column, the value separated by " " means recall value of normal, toe in, toe out, and flat respectively.

Table 3 Experimental results data of static section.

\begin{tabular}{ccccc}
\hline Algorithm & Recall & $\begin{array}{c}\text { Average precision } \\
(\%)\end{array}$ & $\begin{array}{c}\text { Time cost } \\
(\mathrm{ms})\end{array}$ & $\begin{array}{c}\text { Accuracy/time cost } \\
\text { P-value }\end{array}$ \\
\hline LDA+SVMrbf & $0.85 / 0.92 / 1.00 / 0.80$ & 89.39 & 1.10 & $0.698 /<<0.01$ \\
LDA+SVMlin & $0.85 / 0.96 / 1.00 / 0.83$ & 90.90 & 0.50 & -
\end{tabular}




$\begin{array}{ccccc}\text { PCA+SVMlin } & 0.82 / 0.90 / 1.00 / 0.86 & 89.49 & 0.89 & 0.690 /<<0.01 \\ \text { PCA+SVMrbf } & 0.89 / 0.91 / 1.00 / 0.90 & 92.41 & 2.07 & 0.623 /<0.01 \\ & & 89.75 & 0.46 & 0.765 / 0.388\end{array}$

"---" means the P-value of this column is computed based on this algorithm. In the Recall column, the value separated by " " means recall value of normal, toe in, toe out, and flat respectively.

\section{Dynamic Section}
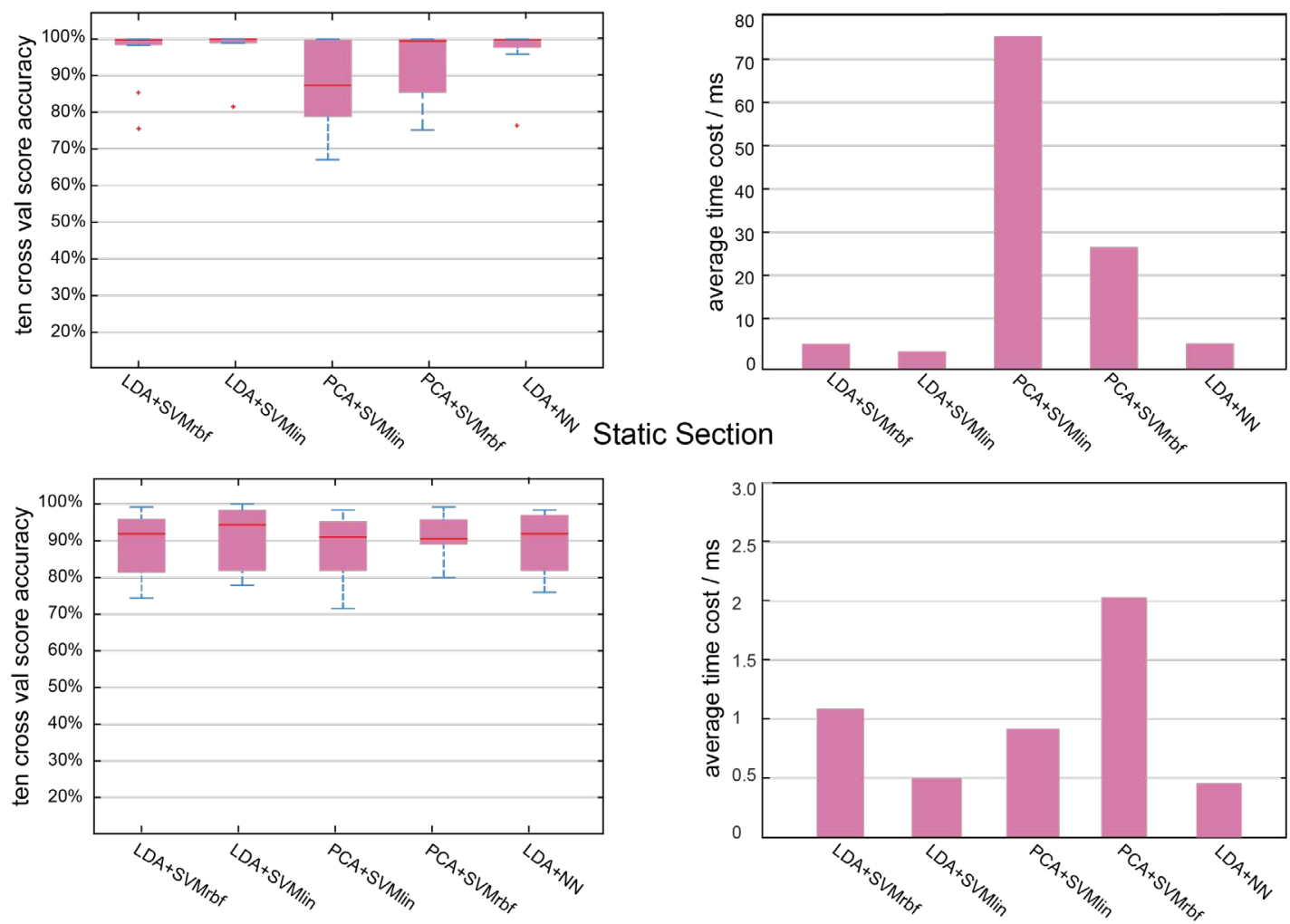

Fig. 7 Accuracy and time-cost results of IGRM in the dynamic and static experiments. The x-axis label means reduction algorithm + classification algorithm. For example, LDA+SVMlin means that IGRM's feature reduction algorithm is LDA, and SVM with linear kernel is its classification algorithm. Here, PCA components are $7(90 \%)$ in the dynamic section and $12(90 \%)$ in the static section. And the dimension of LDA is 3 in both dynamic and static section 
a

\begin{tabular}{|c|c|c|c|c|c|c|c|c|}
\hline \multirow[b]{2}{*}{ labe } & \multicolumn{8}{|c|}{ dynamic } \\
\hline & 0 & 1 & 2 & 3 & 0 & 1 & 2 & 3 \\
\hline 0 & 479.1 & 19 & 0 & 1.9 & 478.9 & 18.8 & 0 & 2.3 \\
\hline 1 & 0.2 & 499.8 & 0 & 0 & 0.2 & 499.7 & 0.1 & 0 \\
\hline 2 & 0 & 17.6 & 482 & 0 & 0 & 11.9 & 488.1 & 0 \\
\hline & 35.7 & 0.8 & 0 & 463.5 & 8.2 & 2.3 & 0.3 & 489.2 \\
\hline & \multicolumn{4}{|c|}{ LDA+SVMrbf } & \multicolumn{4}{|c|}{ LDA+SVMlin } \\
\hline 0 & 338.5 & 4.3 & 14.7 & 142.5 & 452.1 & 19 & 0 & 28.9 \\
\hline 1 & 0 & 500 & 0 & 0 & 0 & 500 & 0 & 0 \\
\hline 2 & 0 & 0 & 500 & 7 & 0 & 0 & 500 & 0 \\
\hline 3 & 87.1 & 4.2 & 2.3 & 406.4 & 58.4 & 11 & 0 & 430.6 \\
\hline & \multicolumn{4}{|c|}{ PCA+SVMlin } & \multirow{5}{*}{\multicolumn{4}{|c|}{ PCA+SVMrbf }} \\
\hline 0 & 480.3 & 16.9 & 0.8 & 2 & & & & \\
\hline 1 & 0.2 & 499.8 & 0 & 0 & & & & \\
\hline 2 & 0 & 17 & 483 & 0 & & & & \\
\hline & 42 & 0 & 0 & 458 & & & & \\
\hline
\end{tabular}

b

\begin{tabular}{|c|c|c|c|c|c|c|c|c|}
\hline \multirow[b]{2}{*}{ label } & \\
\hline & 0 & 1 & 2 & 3 & 0 & 1 & 2 & 3 \\
\hline 0 & 27.9 & 0 & 0 & 4.8 & 27.7 & 0.3 & 0 & 4.7 \\
\hline 1 & 1.8 & 30.2 & 0 & 0.7 & 0.9 & 31.4 & 0 & 0.4 \\
\hline 2 & 0 & 0 & 32.7 & 0 & 0 & 0 & 32.7 & 0 \\
\hline \multirow[t]{2}{*}{3} & 6.5 & 0 & 0 & 26.2 & 5.3 & 0.2 & 0 & 27.2 \\
\hline & \multicolumn{4}{|c|}{ LDA+SVMrbf } & \multicolumn{4}{|c|}{ LDA+SVMlin } \\
\hline 0 & 26.7 & 0 & 0 & 6 & 29.1 & 0 & 0 & 3.6 \\
\hline 1 & 2.5 & 29.3 & 0 & 0.9 & 2.5 & 29.8 & 0 & 0.4 \\
\hline 2 & 0 & 0 & 32.7 & 0 & 0 & 0 & 32.7 & 0 \\
\hline \multirow[t]{2}{*}{3} & 4.5 & 0 & 0 & 28.2 & 3.3 & 0 & 0 & 29.4 \\
\hline & \multicolumn{4}{|c|}{ PCA+SVMlin } & \multirow{5}{*}{\multicolumn{4}{|c|}{ PCA+SVMrbf }} \\
\hline 0 & 26.7 & 0.7 & 0 & 5.3 & & & & \\
\hline 1 & 1 & 31.1 & 0 & 0.6 & & & & \\
\hline 2 & 0 & 0 & 32.7 & 0 & & & & \\
\hline 3 & 5.8 & 0.5 & 0 & 26.4 & & & & \\
\hline
\end{tabular}

Fig. 8 Average confusion matrix of algorithm results in 10-fold cross-validation of a dynamic and $\mathbf{b}$ static sections. Labels $0,1,2$, and 3 represent normal, toe in, toe out, and flat, respectively. The labels on the row are predicted labels and those on the column are actual labels; the samples number at the row label $i$ and the column label $\mathrm{j}$ means the average number of times instances of class $\mathrm{i}$ are classified as class $\mathrm{j}$ in the 10 -fold procedure

According to Table 2, in the dynamic section, LDA+SVMlin reaches the highest average accuracy, $97.79 \%$. In the term of accuracy, independent t-test shows a significant difference between $\mathrm{LDA}+\mathrm{SVMlin}$ and $\mathrm{PCA}+\mathrm{SVMlin}(\mathrm{P}-\mathrm{value}=0.007)$. There is no significant accuracy difference among LDA+SVMrbf, PCA+SVMrbf, and LDA+NN. However, LDA+SVMlin, 4.40ms per 2000 samples, has a significant smaller time cost than LDA+SVMrbf (P-value $<<0.01), \mathrm{PCA}+\mathrm{SVM}$ in (Pvalue $<<0.01$ ), and PCA+SVMrbf (P-value $<<0.01)$. PCA+SVMlin has the largest time cost (P-value $<<0.01), 75.2 \mathrm{~ms}$ per 2000 samples. In the static section, PCA+SVMrbf reaches the highest average accuracy, $92.41 \%$. However, PCA+SVMrbf has a significantly larger time cost than others (P-value $<<0.01), 2.07 \mathrm{~ms}$ per 130 samples. Compared with other algorithms, LDA+SVMlin has no significant difference with other algorithms in terms of accuracy but it has a significantly smaller time cost (Pvalue $<<0.01)$ than LDA+SVMrbf, PCA+SVMlin, and PCA+SVMrbf.

Taking precision, time cost, and statistical analysis into consideration, LDA+SVMlin and $\mathrm{LDA}+\mathrm{NN}$ are excellent classifiers in both dynamic and static sections.

\section{Discussion}


The results in Fig. 7 prove the feasibility, robustness, and high average accuracy of the proposed approach. All of the IGRMs have been identified with a practically applicable degree of average accuracy either in the dynamic or static section.

From the experimental results above, LDA+SVMlin (97.79\% in average precision), LDA+NN (97.38\%) are excellent classifiers in both dynamic and static sections. An independent t-test shows no significant difference between LDA $+\mathrm{NN}$ and LDA + SVMlin in terms of accuracy $(\mathrm{P}-\mathrm{value}=0.702$ in the dynamic section, $\mathrm{P}$-value $=0.765$ in the static section) and time cost $(\mathrm{P}$-value $=0.064$ in the dynamic section, $\mathrm{P}=0.388$ in the static section). However, based on the good interpretability of SVM, a combination of LDA and SVM with linear kernel is our first choice.

The accuracy distribution of the IGRMs shows that LDA is much better than PCA in pathological gait recognition. Results show LDA+SVMrbf and LDA+SVMlin have a significantly smaller variance than PCA+SVMrbf and PCA+SVMlin in the dynamic section, respectively. However, in the static section, the accuracy of LDA+SVMrbf and PCA+SVMrbf, LDA+SVMlin, and PCA+SVMlin has no significant difference. We infer it might be caused by the fact that plantar pressure data in the dynamic section is more complex and efficient than those in the static section. Besides, LDA has higher efficiency in terms of time cost. Results show that PCA+SVM is approximately two to five times the time cost of the LDA+SVM.

According to our prior knowledge, plantar pressure in the static situation have more information about foot types than that in the dynamic situation. More interestingly, we found that the IGRMs in the dynamic section have higher average accuracy than those of the static section. Combined with the corresponding pressure distribution, it can be inferred that children may act more naturally in the dynamic section than in the static section. Regarding pre-experiment sections I and II, the walking gestures of children more closely resemble an actual situation, while in the static section children are prone to stand normally deliberately when asked to stand still, which causes a reduction of accuracy and large variance. Meanwhile, according to doctors' judgments, it is quite difficult to judge toe in and toe out in the static section unless it is in a serious stage.

\section{Conclusions}


In this paper, an effective IGRM for pathological gait recognition with dimensional reduction and a classification algorithm is put forward. Also, a low-cost and wearable PGRS with an $8 \times 8$ pressure sensor array was built. The experimental results show that the proposed IGRM (LDA+SVMlin) has both high accuracy and low prediction time cost in the dynamic section, that is, $97.79 \%$ average accuracy and a 4.4 ms prediction time per 2000 samples, while in the static section $90.90 \%$ average accuracy and an $0.5 \mathrm{~ms}$ prediction time per 130 samples were realized. Another phenomenon found in the experiments is that pathological gait is detected more effectively in the dynamic section since children act more naturally in walking than just standing.

In conclusion, a low-cost PGRS has been verified and realize feasibility, highly average precision, and good real-time performance of gait recognition. Furthermore, the experimental results reveal the potential for the computer supervision of non-pathological and pathological gaits in the plantarpressure patterns of children and for providing feedback in the application of gait-abnormality rectification.

In this study, the implementation of the IGRM is mainly done via a GUI program on a computer. Thus, a PC is still the indispensable device used in pathological gait recognition. In the future, all of the PGRS components should be integrated into an embedded, wearable system to constitute a more powerful, practical PGRS. Besides, more subjects must be involved, and more plantar-pressure data should be collected to build a pathological dataset for more complex pathological gait research.

\section{Methods}

To deal with the gait data with high dimensionality, temporal dependence, high variability, correlations between curves, and nonlinear relationships [4], the proposed gait-recognition algorithm has three steps: feature extraction, feature reduction, and classification. Fig. 9 shows an overview of the abnormal gait-recognition algorithm and corresponding gait patterns. Firstly, the data is transformed from time domain to orthogonal domain to handle the gait's temporal dependence and get data features. Here, several transformations can be used, for example, fast Walsh transform (FWT), discrete cosine transform (DCT), and fast Fourier transform (FFT), among which FFT, used in the proposed IGRM, is the most common and effective one. Later, feature combination and feature 
reduction algorithms are done to make gait-data dimensionality lower to apply it in real-time situations. Finally, to handle the high-variability problem, correlations between curves, and non-linear relationships, a robust classification algorithm is used.

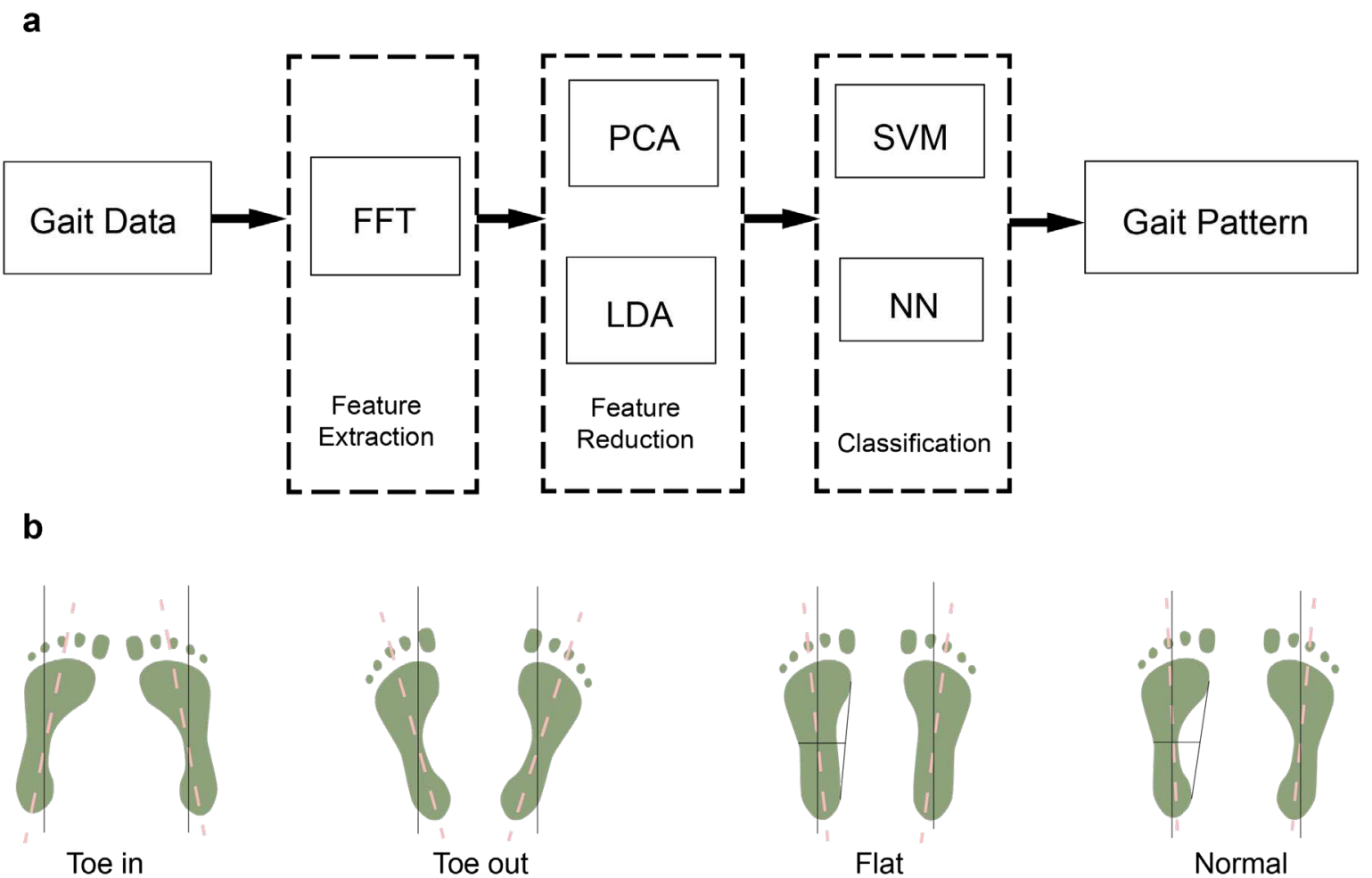

Fig. 9 a Diagram of the gait-recognition algorithm. b Gait pattern for classification - toe in, toe out, flat and normal

\section{Gait Feature Extraction from Gait Cycle}

In the dynamic situation where subjects walk naturally on the level ground wearing PGRS, the detailed gait feature-extraction process is shown in Fig. 10. For length- $L$ data, the $k$ th feature of the FFT $S(k)$ can be obtained through

$$
S(k)=\sum_{n=0}^{L-1} t(n) W^{k n}
$$

where $\mathrm{k}$ is the integer between 0 (included) and L-1 (included); $W$ is $e^{-j 2 \pi / L} ; n$ is the time series and $t(n)$ represents the data value at time $n$.

Simple data augmentation with the sliding window method is used to make a proper classification on the assumption that the stochastic process related to the features is stationary over the window 
interval. We find that if a window interval $L$ is chosen wide enough to include multiple gait periods, then the assumption can be acceptable. Consequently, in this step, the temporal dependence is removed through FFT. In this paper, the sliding window is Hanning window with a width of 512 sample intervals $(50 \mathrm{~Hz}$ sample frequency, corresponding to about 10 gait periods). A 512-length vector $S(k)$ can be got by transforming the plantar-pressure data in the sliding window to frequency domain using FFT. The vector denotes the energy information distribution on the frequency domain of a pressure sensor block during walking. Therefore, vectors $S_{i, j}(k)$, where $i=1 \cdots 8$ and $j=1 \cdots 8$, including all $8 \times 8$ sensor blocks' frequency information can describe the entire foot energy information distribution. Since the experiments show that the major information of children's gait data is between 0 and $10 \mathrm{~Hz}$, gait data are divided into five groups which are 0 (exclude) $-2 \mathrm{~Hz}, 2$ (exclude) $-4 \mathrm{~Hz}, 4$ (exclude) $-6 \mathrm{~Hz}, 6$ (exclude) $-8 \mathrm{~Hz}$, and 8 (exclude) - $10 \mathrm{~Hz}$. This division of groups is optimized by numerous simulations. Summing all of the amplitude of frequency components in each group, the 512-length vector $S(k)$ is transformed to a five-element feature vector. To denote the information of the entire foot, 30 five-element feature vectors from 30 sensors are joined together, obtaining a 150-element vector as a training sample. Note that bodyweight is different for different children, the $0 \mathrm{~Hz}$ pressure information is excluded and the 150 -element training sample is normalized to a unit vector to eliminate the weight information influence when classifying the foot types.

In the static situation where subjects stand naturally on the level ground wearing PGRS, the $0 \mathrm{~Hz}$ pressure information is kept only and the feature number of a plantar pressure sensor is no longer five but one. The final training sample for gait recognition becomes a 30 -element vector. Normalization is also used to vanish the influence of the bodyweight of the subjects. 
b

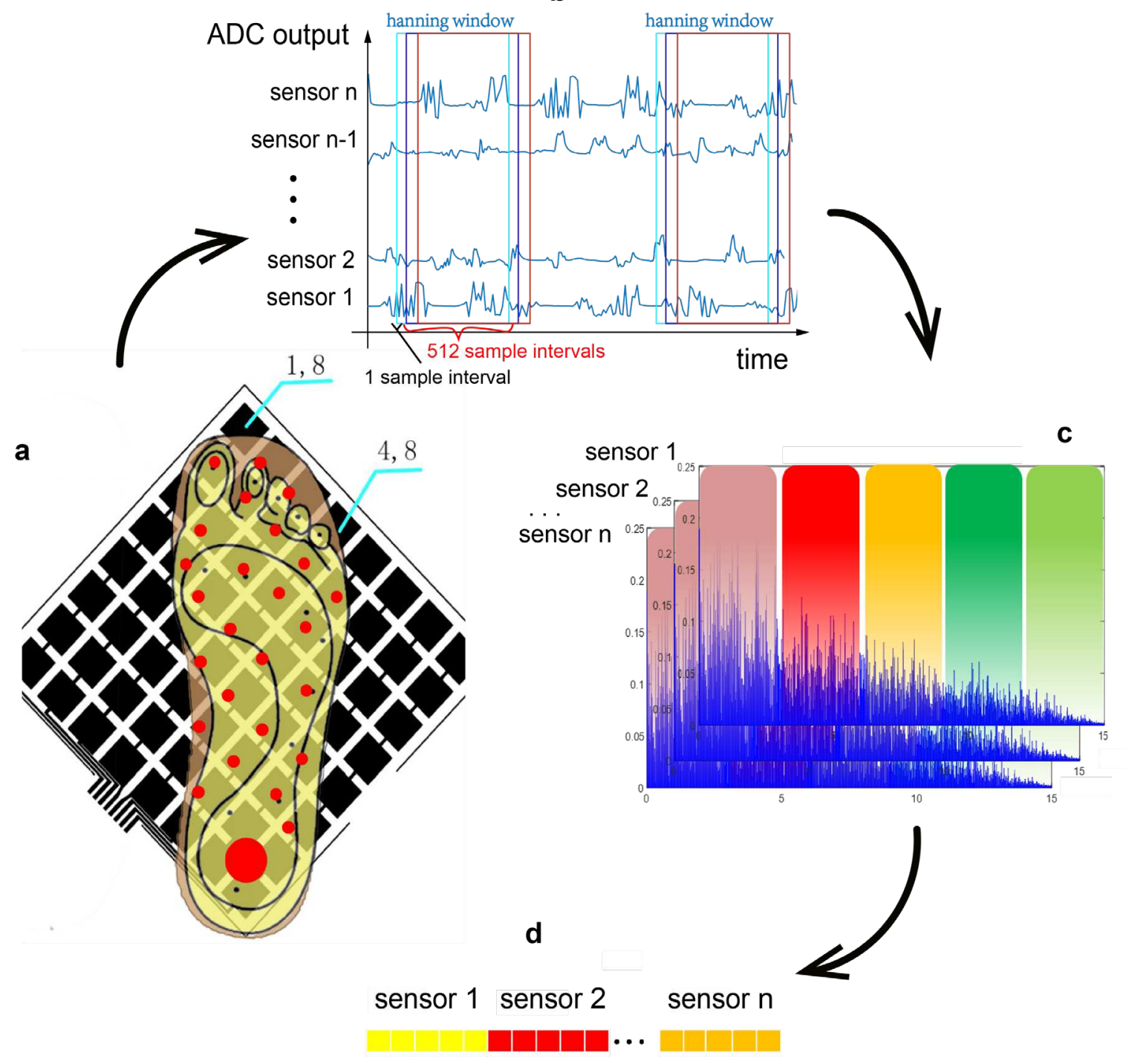

Fig. 10 The procedure of gait feature extraction from gait cycle. a 30 sensor blocks with the red mark are selected for feature extraction. b Sliding window method (Hanning window with 512-sample intervals width) is applied to all $\mathrm{n}$ sensor blocks and transformed to frequency domain later. $\mathbf{c}$ Dividing five frequency bands from FFT frequency spectrum, 0 (exclude) - $2 \mathrm{~Hz}, 2$ (exclude) $-4 \mathrm{~Hz}, 4$ (exclude) $-6 \mathrm{~Hz}, 6$ (exclude) $-8 \mathrm{~Hz}$, and 8 (exclude) $-10 \mathrm{~Hz}$, and summing all the frequent value in each frequency band to generate a five-elements vector for each sensor. d Joining all the five-element vector from each sensor together and normalizing to a 150 element unit vector.

\section{Feature Reduction}

In this step, plantar-pressure information is further compressed and keeps effectiveness at the same time. As a quite effective and common unsupervised method in signal processing, PCA keeps 
the variance of the original data during the dimension reduction. However, PCA may cause a mix-up of different-label data in some situations, as Fig. 11a shows. In these situations, another supervised method, LDA, is more efficient. LDA maximizes the average differences among class projections while minimizing average projections of each class (intraclass) after feature reduction. In mathematics, the main idea of LDA can be described as maximizing $J(W)$

$$
\max J(W)=\frac{\sum_{i} \tilde{S}_{B(i)}}{\sum_{j} \tilde{S}_{W(j)}}=\frac{\sum_{i} \mathbf{W}^{T} S_{B(i)} \mathbf{W}}{\sum_{j} \mathbf{W}^{T} S_{W(j)} \mathbf{W}},
$$

where $S_{B}$ and $S_{W}$ are the dispersion between two different classes and within a class, respectively; i and $\mathrm{j}$ represent the class number; symbol $\sim$ represents the variable after dimensional reduction and $\mathbf{W}^{T}$ is defined as the corresponding transformation matrix.

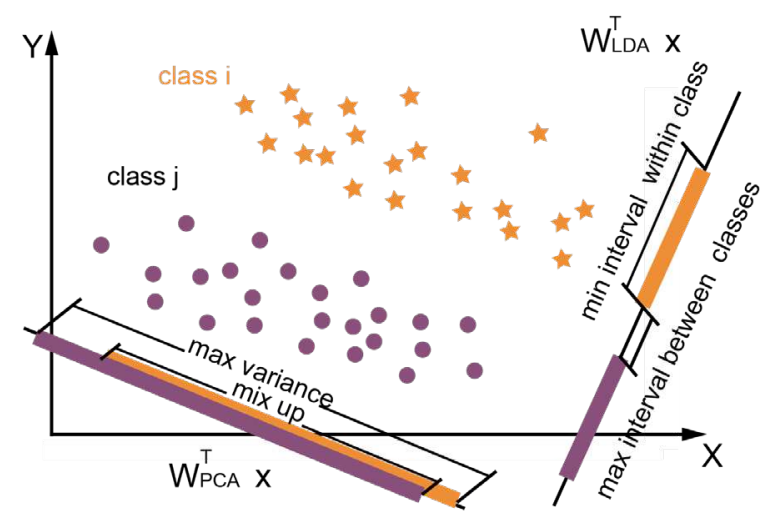

a

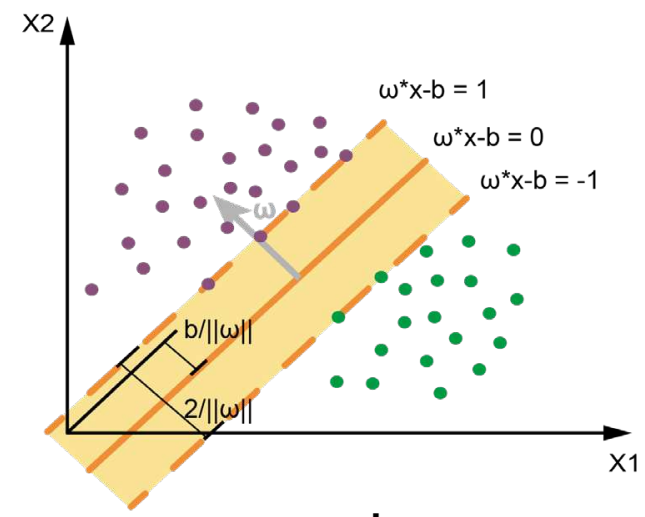

b

Fig. 11 a LDA and PCA algorithm performance in classification in the specific situation. In this situation, LDA's performance is better than PCA's. b SVM classification. The main idea of the SVM is projecting data points into a higher dimensional space, specified by a kernel function, and computing a maximum-margin hyperplane decision surface that separates the two classes

\section{Gait-Classification Model}

Our goal in this step is to classify the gaits into different classes, i.e., toe in, toe out, flat, and normal according to the feature vectors. Many algorithms in the machine learning field can be used for binary classification problems. In this paper, NN and SVM are used to perform the gait-classification function. 
As many researchers have proved, SVM can work very well for multi-dimensional data $[32,33]$. More importantly, small computation is needed for the final decision function of SVM which is comprised of only a few support vectors. As shown in Fig. $11 \mathrm{~b}$, it obtains a hyperplane, $\boldsymbol{\omega}^{\mathrm{T}} \mathbf{x}+b=0$, to classify two classes by maximizing the margin to the closest data from two classes separately. The decision function can be described as

$$
\left\{\begin{array}{l}
f(\mathbf{x})=\boldsymbol{\omega}^{\mathbf{T}} \mathbf{x}+b \\
y=\operatorname{sign}(f(\mathbf{x}))
\end{array}\right.
$$

where $\mathbf{x}$ is the feature vector, $\boldsymbol{\omega}$ is the normal vector to the hyperplane, $\frac{b}{\|\boldsymbol{\omega}\|}$ determines the offset of the hyperplane, and $y$ are either 1 or -1 , each indicating the class to which the feature vector belongs. A linear SVM's goal is to minimize the risk function

$$
\begin{aligned}
& \underset{\omega, b}{\operatorname{Minimize}} \frac{1}{2} \boldsymbol{\omega}^{\mathbf{T}} \boldsymbol{\omega}+C \sum_{i=1}^{N} \xi_{i} \\
& \text { Subject to }\left\{\begin{array}{l}
\xi_{i} \geq 0 \\
y_{i}\left(\boldsymbol{\omega}^{\mathbf{T}} \mathbf{x}+b\right) \geq 1-\xi_{i}
\end{array}\right.
\end{aligned}
$$

where the term $\boldsymbol{\omega}^{\mathbf{T}} \boldsymbol{\omega}$ is called the regularization term and $C \sum_{i=1}^{N} \xi_{i}$ is empirical tolerance [34]. Empirical tolerance is not only used to remove data noise, but also to deal with data non-linearly separable. To obtain the linear inseparable classification model, the penalty parameter $C$ calculates the penalties for errors by determining the trade-off between the empirical tolerance and regularized term. The larger $C$ is, the stronger penalties are assigned to errors.

To solve the non-linear classification problem effectively, a kernel function is introduced to the decision function

$$
f\left(\mathbf{x}^{\mathbf{T}} \mathbf{x}\right) \stackrel{\text { kernel }}{\longrightarrow} f\left(\phi\left(\mathbf{x}_{\mathbf{i}}, \mathbf{x}\right)\right),
$$

where $\phi\left(\mathbf{x}_{\mathbf{i}}, \mathbf{x}\right)$ is a kernel function that maps $\mathbf{x}$ space to a higher-dimensional space so that a hyperplane can be found to classify samples. In this paper, linear or RBF kernel is used in our classification model. To solve this formulation, SVM can be trained by Platt's sequential minimal optimization (SMO) algorithm [35]. 
$\mathrm{NN}$ is a network of neurons and the connections of biological neurons are modeled as weights. Training the weight using the backpropagation algorithm, NN can learn the mapping relationship between input and output. Regardless of its powerful ability for solving nonlinear problems, $\mathrm{NN}$ is a black box comparing with explainable and intuitive SVM.

\section{Declarations}

\section{Ethics approval and consent to participate}

The study was performed following the principles outlined in the Helsinki Declaration and it was approved by Clinical Research Ethics Committee. All procedures were carried out with the adequate understanding and written informed consent of each subject.

\section{Consent to publication}

The participants acknowledged their consent to publish the acquired data.

\section{Availability of data and materials}

The datasets used and/or analyzed during the current study are available from the corresponding author on reasonable request.

\section{Competing interests}

The authors declare that they have no competing interests.

\section{Funding}

This research is supported by both National Natural Science Foundation of China (Grant No. 51805469) and Science and technology plan project of drug regulatory system of Zhejiang province (No. 2020016).

\section{Authors' contributions}

LX, FW, and WY carried out the concept and design of the study and interpreted the data. LX performed the statistical analysis and signal processing. LX, JC, and WY participated in the 
acquisition of gait data. LX drafted the manuscript. JC, YC, WY, and CY provided critical revision of the manuscript for important intellectual content. FW, WY, and CY conceived of the study and obtained funding. CY provided administrative, technical, and material support for the study. CY supervised the study. All authors read and approved the final manuscript.

\section{Acknowledgments}

We are very grateful to the children and parents who generously gave their time to assist with this research.

\section{Reference}

[1] Figueiredo J, Santos CP, Moreno JC. Automatic recognition of gait patterns in human motor disorders using machine learning: A review. Medical Engineering \& Physics 2018;53:1-12. https://doi.org/10.1016/j.medengphy.2017.12.006.

[2] Titianova EB, Mateev PS, Tarkka IM. Footprint analysis of gait using a pressure sensor system. Journal of Electromyography and Kinesiology 2004;14:275-81. https://doi.org/10.1016/S10506411(03)00077-4.

[3] Salarian A, Russmann H, Vingerhoets FJG, Dehollain C, Blanc Y, Burkhard PR, et al. Gait Assessment in Parkinson's Disease: Toward an Ambulatory System for Long-Term Monitoring. IEEE Trans Biomed Eng 2004;51:1434-43. https://doi.org/10.1109/TBME.2004.827933.

[4] Chau T. A review of analytical techniques for gait data. Part 1: Fuzzy, statistical and fractal methods. Gait Posture 2001;13:49-66. https://doi.org/10.1016/s0966-6362(00)00094-1.

[5] E D, B T, A M. An Automated Classification of Pathological Gait Using Unobtrusive Sensing Technology. IEEE Trans Neural Syst Rehabil Eng 2017;25:2336-46. https://doi.org/10.1109/tnsre.2017.2736939.

[6] Ortells J, Herrero-Ezquerro MT, Mollineda RA. Vision-based gait impairment analysis for aided diagnosis. Med Biol Eng Comput 2018;56:1553-64. https://doi.org/10.1007/s11517-018-1795-2. 
[7] Zakaria NK. ASD Children Gait Classification Based On Principal Component Analysis and Linear Discriminant Analysis. 2020;8:2438-45. https://doi.org/10.30534/ijeter/2020/38862020.

[8] Chen S-W, Lin S-H, Liao L-D, Lai H-Y, Pei Y-C, Kuo T-S, et al. Quantification and recognition of parkinsonian gait from monocular video imaging using kernel-based principal component analysis. BioMed Eng OnLine 2011;10:99. https://doi.org/10.1186/1475-925X-10-99.

[9] Mei Z, Ivanov K, Zhao G, Li H, Wang L. An explorative investigation of functional differences in plantar center of pressure of four foot types using sample entropy method. Med Biol Eng Comput 2017;55:537-48. https://doi.org/10.1007/s11517-016-1532-7.

[10] Mei Z, Zhao G, Ivanov K, Guo Y, Zhu Q, Zhou Y, et al. Sample entropy characteristics of movement for four foot types based on plantar centre of pressure during stance phase. BioMed Eng OnLine 2013;12:101. https://doi.org/10.1186/1475-925X-12-101.

[11] Hs Z, N M, Jg W, Wj T, P B-R, Jj W. An umbilical data-acquisition system for measuring pressures between the foot and shoe. IEEE Trans Biomed Eng 1990;37:908-11. https://doi.org/10.1109/10.58601.

[12] Zhu HS, Wertsch JJ, Harris GF, Loftsgaarden JD, Price MB. Foot pressure distribution during walking and shuffling. Arch Phys Med Rehabil 1991;72:390-7.

[13] Lin Y-C, Lin Y-T. Human recognition based on plantar pressure patterns during gait. J Mech Med Biol 2013;13:1350039. https://doi.org/10.1142/S0219519413500395.

[14] Saono ES, Bumpus T, Zeigler S, Marocco S. Classification of plantar pressure and heel acceleration patterns using neural networks. vol. 5, IEEE; 2005, p. 3007-10. https://doi.org/10.1109/IJCNN.2005.1556404.

[15] Meng Chen, Bufu Huang, Yangsheng Xu. Intelligent shoes for abnormal gait detection. 2008 IEEE International Conference on Robotics and Automation, 2008, p. 2019-24. https://doi.org/10.1109/ROBOT.2008.4543503.

[16] Robb J. In-Toeing and Out-Toeing Gait. In: Joseph B, Robb J, Loder RT, Torode I, editors. Paediatric Orthopaedic Diagnosis: Asking the Right Questions, Springer India; 2015, p. 207-20. https://doi.org/10.1007/978-81-322-2392-4_21. 
[17] Thackeray C, Beeson P. Is in-toeing gait a developmental stage? The Foot 1996;6:19-24. https://doi.org/10.1016/S0958-2592(96)90055-3.

[18] Thackeray C, Beeson P. In-toeing gait in children. A review of the literature. The Foot 1996;6:14. https://doi.org/10.1016/S0958-2592(96)90051-6.

[19] Jacobs B. Toe walking, flat feet and bow legs, in-toeing and out-toeing. Paediatrics and Child Health 2010;20:221-4. https://doi.org/10.1016/j.paed.2010.03.001.

[20] García-Rodríguez A, Martín-Jiménez F, Carnero-Varo M, Gómez-Gracia E, Gómez-Aracena J, Fernández-Crehuet J. Flexible flat feet in children: a real problem? Pediatrics 1999;103:e84. https://doi.org/10.1542/peds.103.6.e84.

[21] Pfeiffer M, Kotz R, Ledl T, Hauser G, Sluga M. Prevalence of Flat Foot in Preschool-Aged Children. Pediatrics 2006;118:634-9. https://doi.org/10.1542/peds.2005-2126.

[22] Yh L, Jc L. Intoeing gait in children. Hong Kong Med J 1999;5:360-6.

[23] Wren TAL, Rethlefsen S, Kay RM. Prevalence of specific gait abnormalities in children with cerebral palsy: influence of cerebral palsy subtype, age, and previous surgery. J Pediatr Orthop 2005;25:79-83. https://doi.org/10.1097/00004694-200501000-00018.

[24] Rosenbaum D. Foot loading patterns can be changed by deliberately walking with in-toeing or out-toeing gait modifications. Gait \& Posture 2013;38:1067-9. https://doi.org/10.1016/j.gaitpost.2013.04.001.

[25] S Y-L, H J-L, X F-Q. Survey on the occurrence of flatfoot among 1001 children aged 3-12 years old in CangZhou city. Modern Preventive Medicine 2008.

[26] Urry S. Plantar pressure-measurement sensors. Meas Sci Technol 1999;10:R16-32. https://doi.org/10.1088/0957-0233/10/1/017.

[27] Sundaram S, Kellnhofer P, Li Y, Zhu J-Y, Torralba A, Matusik W. Learning the signatures of the human grasp using a scalable tactile glove. Nature 2019;569:698-702. https://doi.org/10.1038/s41586-019-1234-z.

[28] D'Alessio T. Measurement errors in the scanning of piezoresistive sensors arrays. Sensors and Actuators A: Physical 1999;72:71-6. https://doi.org/10.1016/S0924-4247(98)00204-0. 
[29] Speeter TH. A Tactile Sensing System for Robotic Manipulation. The International Journal of Robotics Research 1990;9:25-36. https://doi.org/10.1177/027836499000900603.

[30] Casiez G, Roussel N, Vogel D. 1€ Filter: A Simple Speed-based Low-pass Filter for Noisy Input in Interactive Systems, ACM; 2012, p. 2527. https://doi.org/10.1145/2207676.2208639.

[31] Qihang W, Wenlian L, Zuyun F, Shibi L. A new system for foot pressure measurement and gait analysis 2000;19:32-40.

[32] Steinwart I, Christmann A. Support Vector Machines. 1st ed. Springer Publishing Company, Incorporated; 2008.

[33] Deisenroth MP, Faisal AA, Ong CS. Mathematics for Machine Learning. Cambridge ; New York, NY: 2020 .

[34] Chen M, Huang B, Lee KK, Xu Y. An Intelligent Shoe-Integrated System for Plantar Pressure Measurement. 2006 IEEE International Conference on Robotics and Biomimetics, Kunming, China: IEEE; 2006, p. 416-21. https://doi.org/10.1109/ROBIO.2006.340213.

[35] Platt JC. Fast training of support vector machines using sequential minimal optimization. Advances in Kernel Methods: Support Vector Learning 1999:185-208. 


\section{Figures}

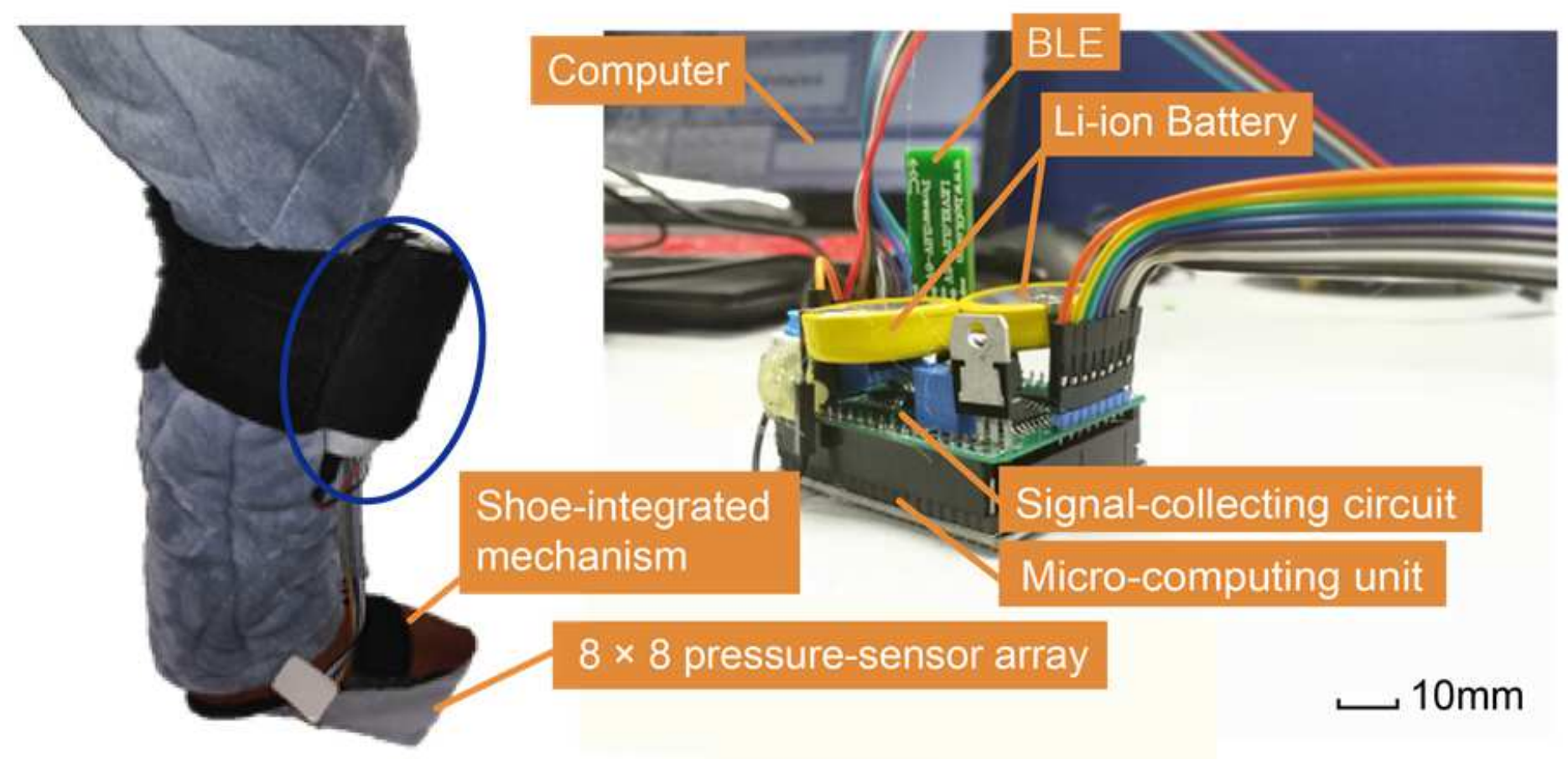

Figure 1

Pathological gait-recognition system. $8 \times 8$ sensor array is under the slipper and controller attached to the child's leg with hook-and-loop fasteners. The control circuit board contains a signal-collecting circuit, a low-energy Bluetooth device (HC-42 with Bluetooth 5.0, HuiCheng Information Technology Co., Ltd., China), STM32F103 controller, and two 4.2-V Li-ion batteries. The signal-collecting circuit operates with 5 $\mathrm{V}$ of power generated by an LM7805 unit (KIA7805AP, three-terminal positive voltage regulator of $5 \mathrm{~V}, \mathrm{KEC}$, China) and the STM32F103 circuitry operates with $3.3 \mathrm{~V}$ generated by an AMS117 unit (low-dropoutvoltage regulator with fixed 3.3 V, Advanced Monolithic Systems, Inc.) 

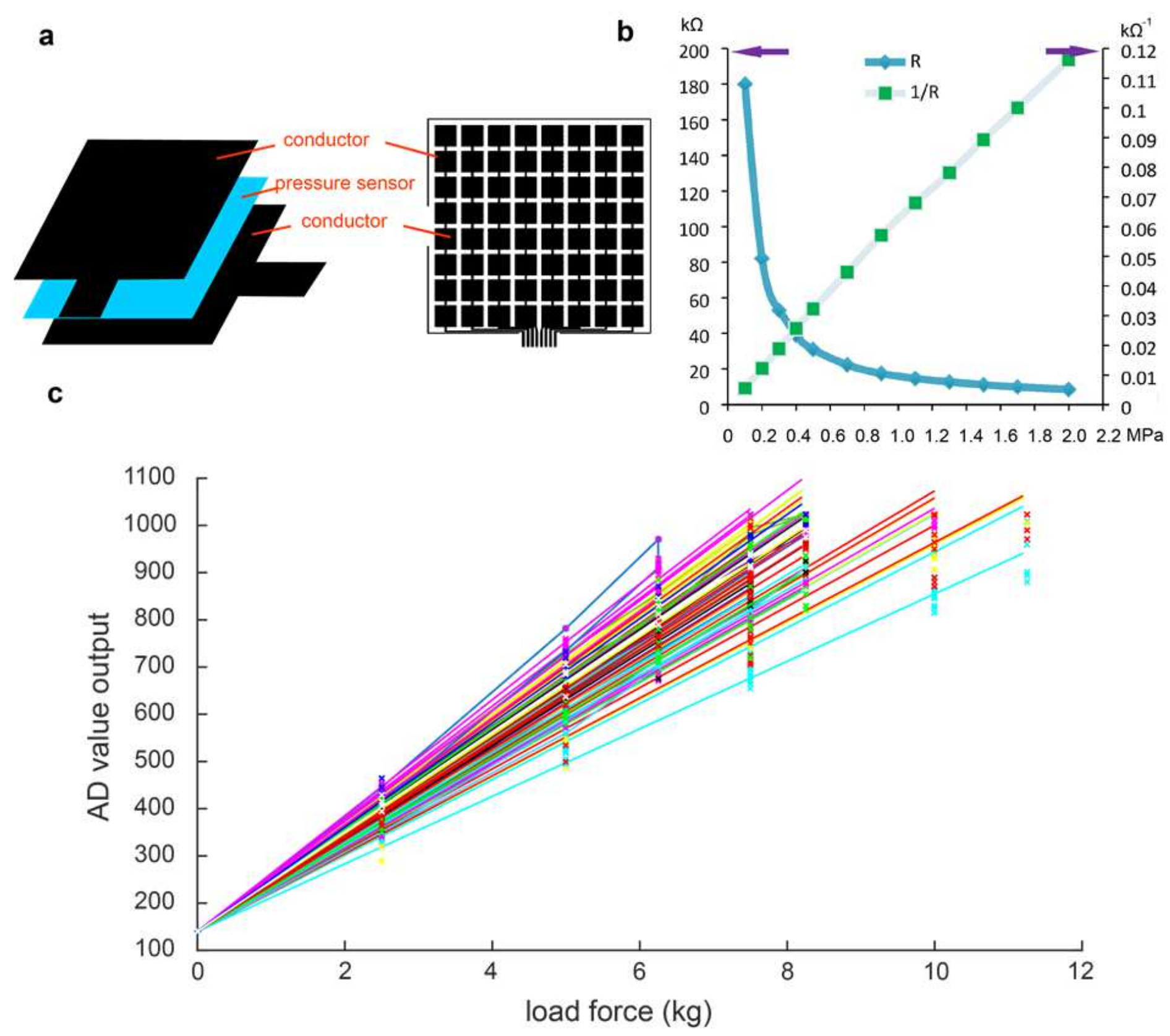

Figure 2

a The three-layer structure of the piezoresistive sensor. b Piezoresistive sensor pressure-resistor characteristic curve. c Sensor calibration 

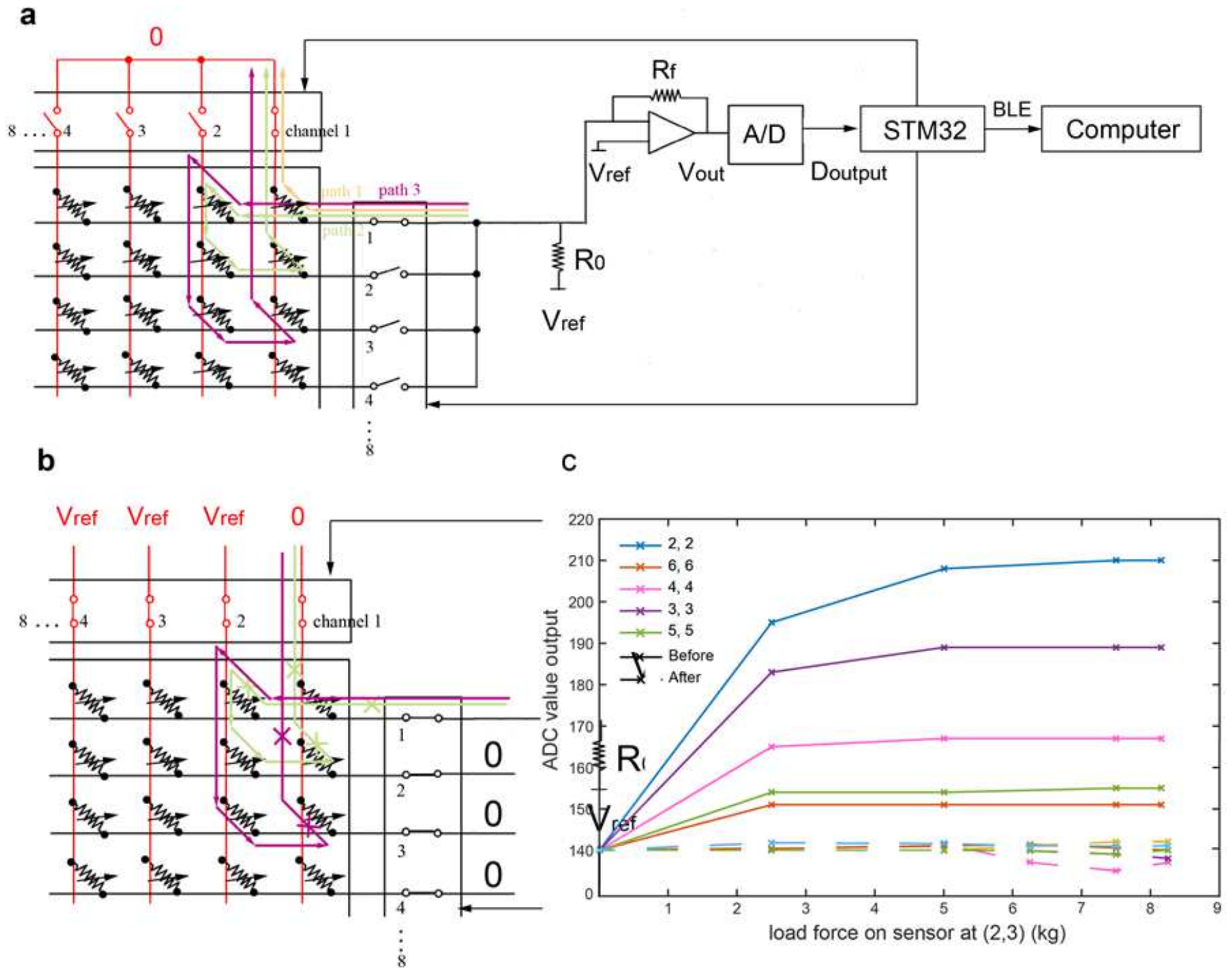

Figure 3

Piezoresistive sensor array scanner electronic schematic. a Schematic before pressure decoupling. A microcontroller is used to select the sampling row and column channel by controlling quad bilateral switch CD4066 chip, while other unselected channels are remaining high resistance. Coupling output is found between sensor blocks as path 1 and path 2 show. b Schematic after pressure decoupling. When a certain row and column channels are selected (row channel 1 and column channel 1 is selected here), we pull down other row channels to ground and pull up other column channels to Vref, such that path 2 and path 3 will be cut off and path 1 will remain. Coupling output between sensor blocks can be eliminated. $c$ The load force on the sensor block at $(2,3)$ has less influence on other sensors' value output after sensor array decoupling. The dotted line represents data after sensor array decoupling and the solid line represents data before sensor array decoupling 


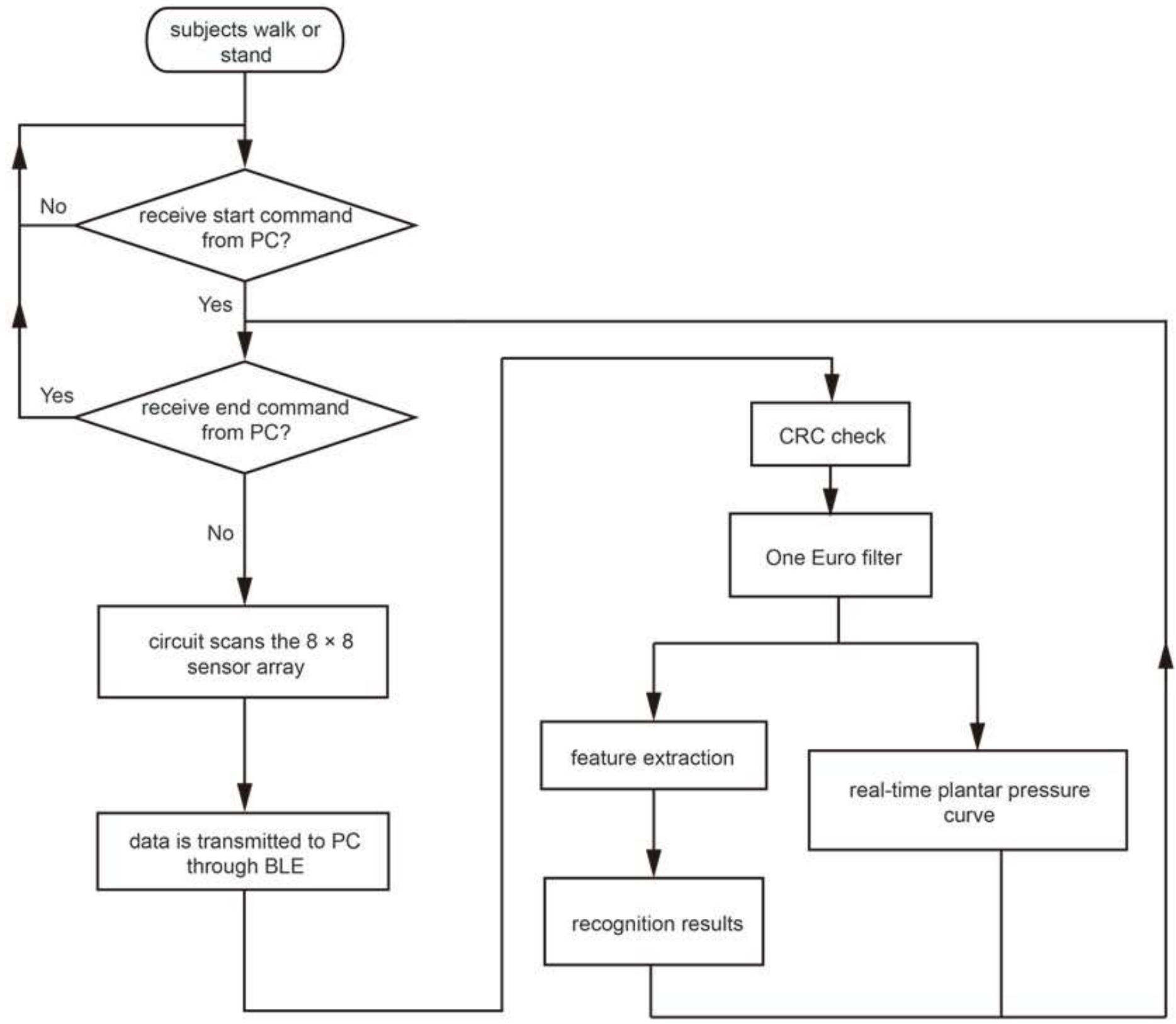

\section{Figure 4}

GUI background flowchart. GUI/PC can receive commands from people in the GUI and use BLE to communicate with the controller system 


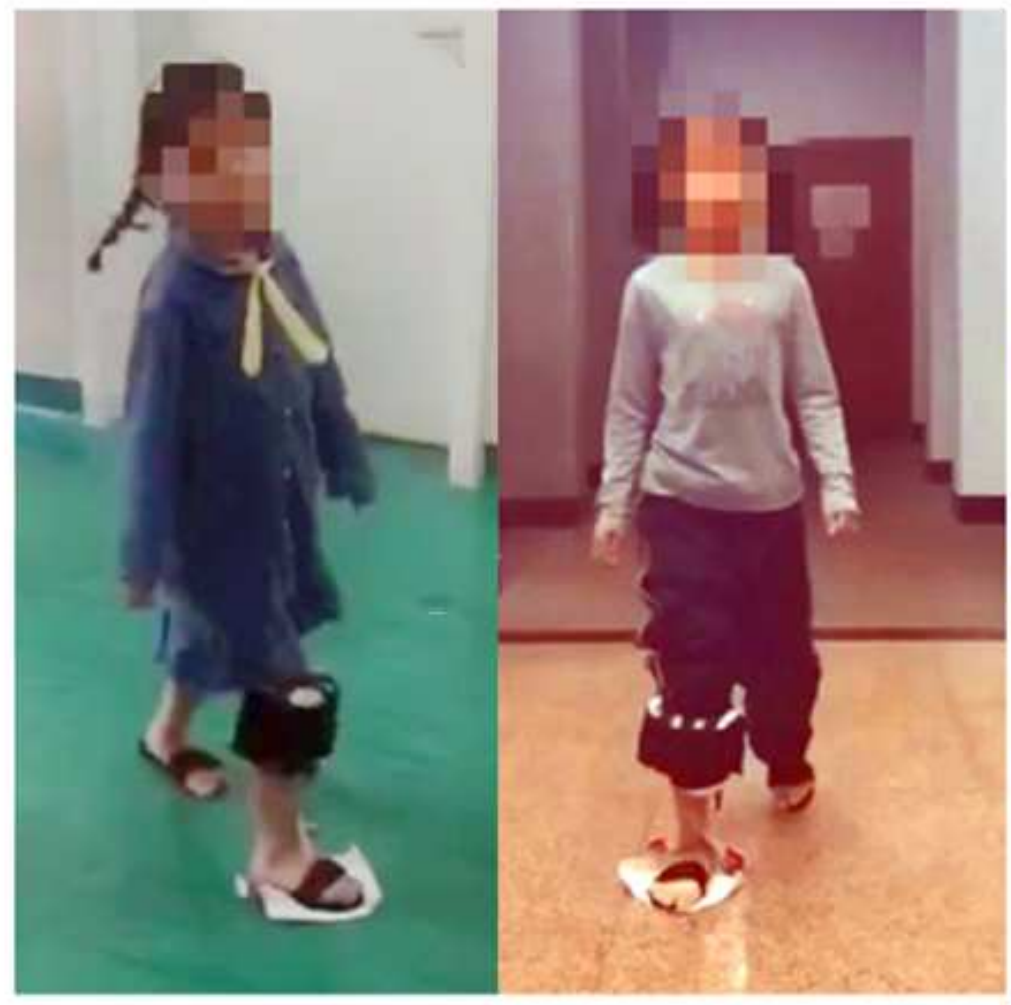

a

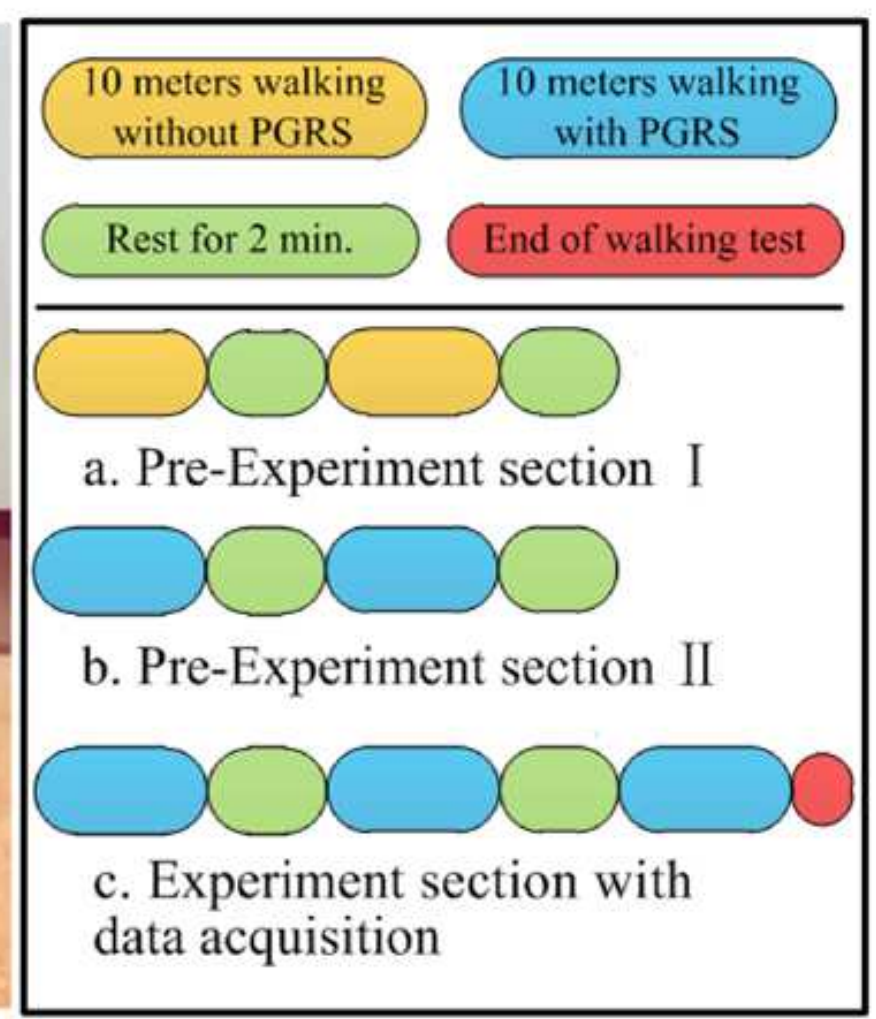

b

Figure 5

Experiment in the dynamic section. a snapshots of subjects. b Experimental procedure of the dynamic section

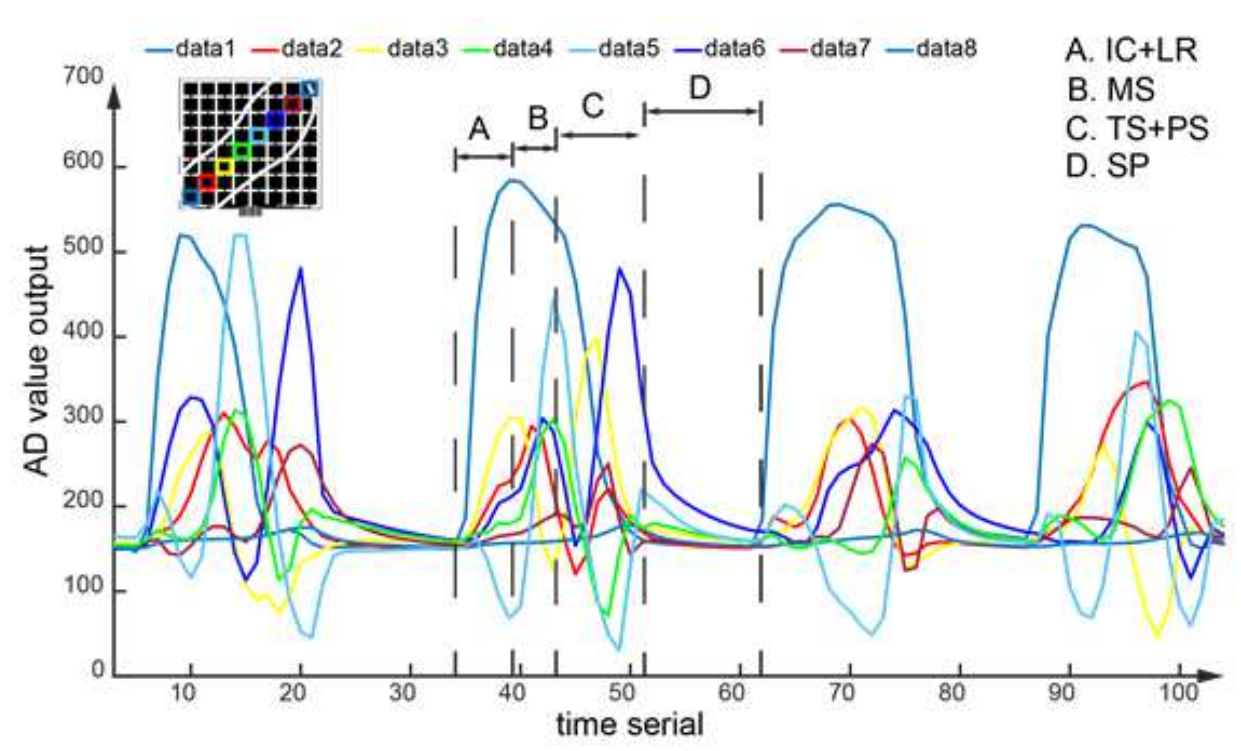

a

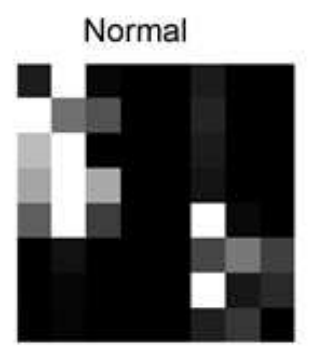

Toe out

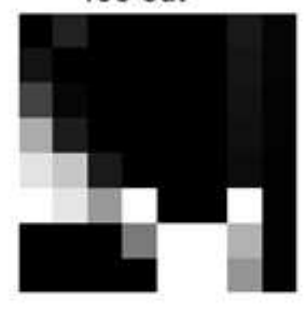

b

Figure 6 
Plantar-pressure data acquisition results. a Curve of plantar pressure with time during level-ground walking in the dynamic-section experiment. b Typical toe in, toe out, and normal foot-pressure distribution in the static-section experiment. The plantar pressure value is expressed in different grey scales. Pure black means zero pressure, pure white means the largest pressure

\section{Dynamic Section}
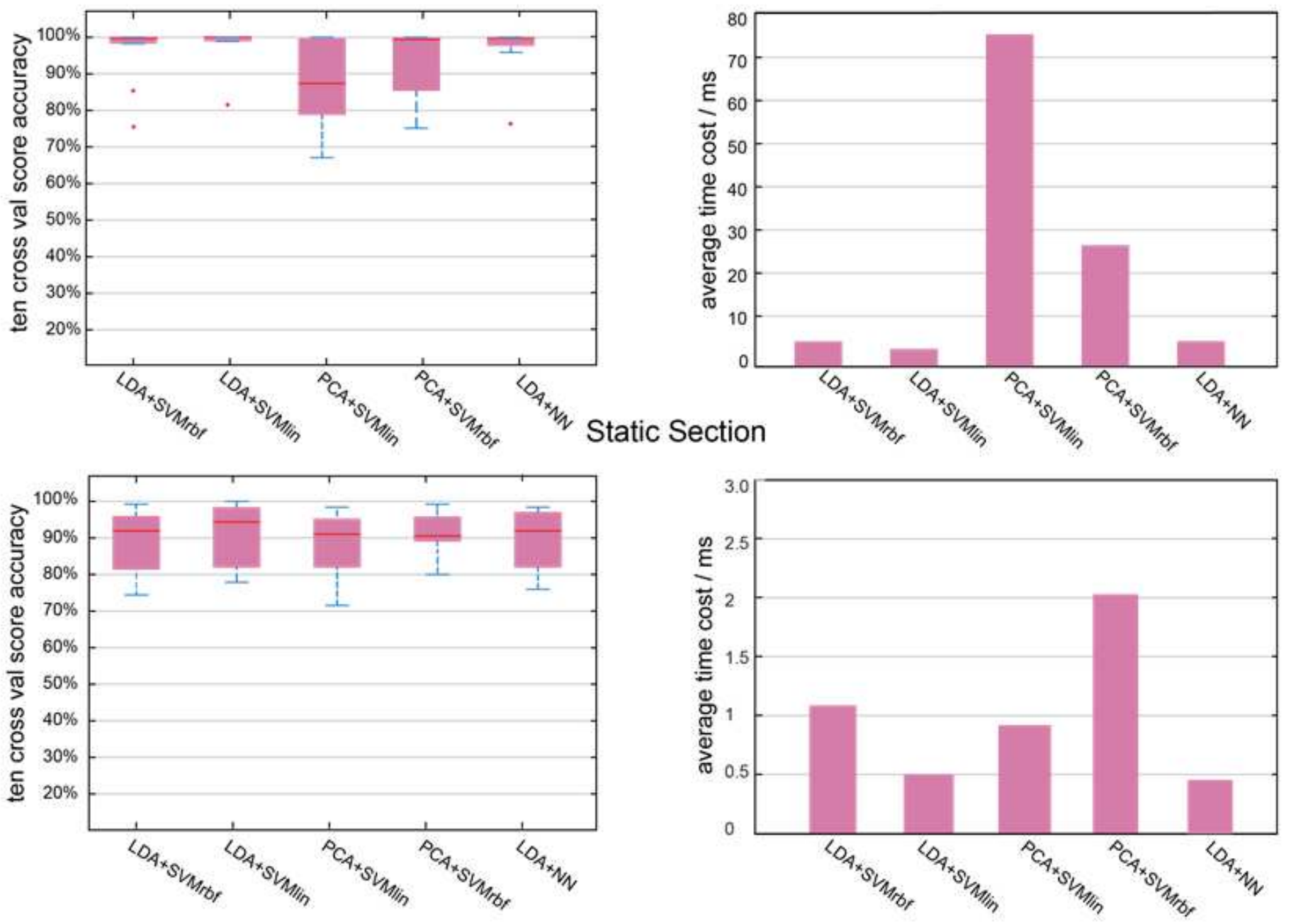

\section{Figure 7}

Accuracy and time-cost results of IGRM in the dynamic and static experiments. The x-axis label means reduction algorithm + classification algorithm. For example, LDA+SVMlin means that IGRM's feature reduction algorithm is LDA, and SVM with linear kernel is its classification algorithm. Here, PCA components are $7(90 \%)$ in the dynamic section and $12(90 \%)$ in the static section. And the dimension of LDA is 3 in both dynamic and static section 
a

\begin{tabular}{|c|c|c|c|c|c|c|c|c|}
\hline label & 0 & 1 & 2 & 3 & 0 & 1 & 2 & 3 \\
\hline 0 & 479.1 & 19 & 0 & 1.9 & 478.9 & 18.8 & 0 & 2.3 \\
\hline 1 & 0.2 & 499.8 & 0 & 0 & 0.2 & 499.7 & 0.1 & 0 \\
\hline 2 & 0 & 17.6 & 482 & 0 & 0 & 11.9 & 488.1 & 0 \\
\hline 3 & 35.7 & 0.8 & 0 & 463.5 & 8.2 & 2.3 & 0.3 & 489.2 \\
\hline & \multicolumn{4}{|c|}{ LDA+SVMrbf } & \multicolumn{4}{|c|}{ LDA+SVMlin } \\
\hline 0 & 338.5 & 4.3 & 14.7 & 142.5 & 452.1 & 19 & 0 & 28.9 \\
\hline 1 & 0 & 500 & 0 & 0 & 0 & 500 & 0 & 0 \\
\hline 2 & 0 & 0 & 500 & 7 & 0 & 0 & 500 & 0 \\
\hline 3 & 87.1 & 4.2 & 2.3 & 406.4 & 58.4 & 11 & 0 & 430.6 \\
\hline & \multicolumn{4}{|c|}{ PCA+SVMlin } & \multirow{5}{*}{\multicolumn{4}{|c|}{ PCA+SVMrbf }} \\
\hline 0 & 480.3 & 16.9 & 0.8 & 2 & & & & \\
\hline 1 & 0.2 & 499.8 & 0 & 0 & & & & \\
\hline 2 & 0 & 17 & 483 & 0 & & & & \\
\hline 3 & 42 & 0 & 0 & 458 & & & & \\
\hline
\end{tabular}

b

static

\begin{tabular}{|c|c|c|c|c|c|c|c|c|}
\hline label & 0 & 1 & 2 & 3 & 0 & 1 & 2 & 3 \\
\hline 0 & 27.9 & 0 & 0 & 4.8 & 27.7 & 0.3 & 0 & 4.7 \\
\hline 1 & 1.8 & 30.2 & 0 & 0.7 & 0.9 & 31.4 & 0 & 0.4 \\
\hline 2 & 0 & 0 & 32.7 & 0 & 0 & 0 & 32.7 & 0 \\
\hline 3 & 6.5 & 0 & 0 & 26.2 & 5.3 & 0.2 & 0 & 27.2 \\
\hline & \multicolumn{4}{|c|}{ LDA+SVMrbf } & \multicolumn{4}{|c|}{ LDA+SVMlin } \\
\hline 0 & 26.7 & 0 & 0 & 6 & 29.1 & 0 & 0 & 3.6 \\
\hline 1 & 2.5 & 29.3 & 0 & 0.9 & 2.5 & 29.8 & 0 & 0.4 \\
\hline 2 & 0 & 0 & 32.7 & 0 & 0 & 0 & 32.7 & 0 \\
\hline 3 & 4.5 & 0 & 0 & 28.2 & 3.3 & 0 & 0 & 29.4 \\
\hline & \multicolumn{4}{|c|}{ PCA+SVMlin } & \multirow{5}{*}{\multicolumn{4}{|c|}{ PCA+SVMrbf }} \\
\hline 0 & 26.7 & 0.7 & 0 & 5.3 & & & & \\
\hline 1 & 1 & 31,1 & 0 & 0.6 & & & & \\
\hline 2 & 0 & 0 & 32.7 & 0 & & & & \\
\hline 3 & 5.8 & 0.5 & 0 & 26.4 & & & & \\
\hline
\end{tabular}

Figure 8

Average confusion matrix of algorithm results in 10-fold cross-validation of a dynamic and b static sections. Labels $0,1,2$, and 3 represent normal, toe in, toe out, and flat, respectively. The labels on the row are predicted labels and those on the column are actual labels; the samples number at the row label $i$ and the column label $\mathrm{j}$ means the average number of times instances of class $\mathrm{i}$ are classified as class $\mathrm{j}$ in the 10-fold procedure

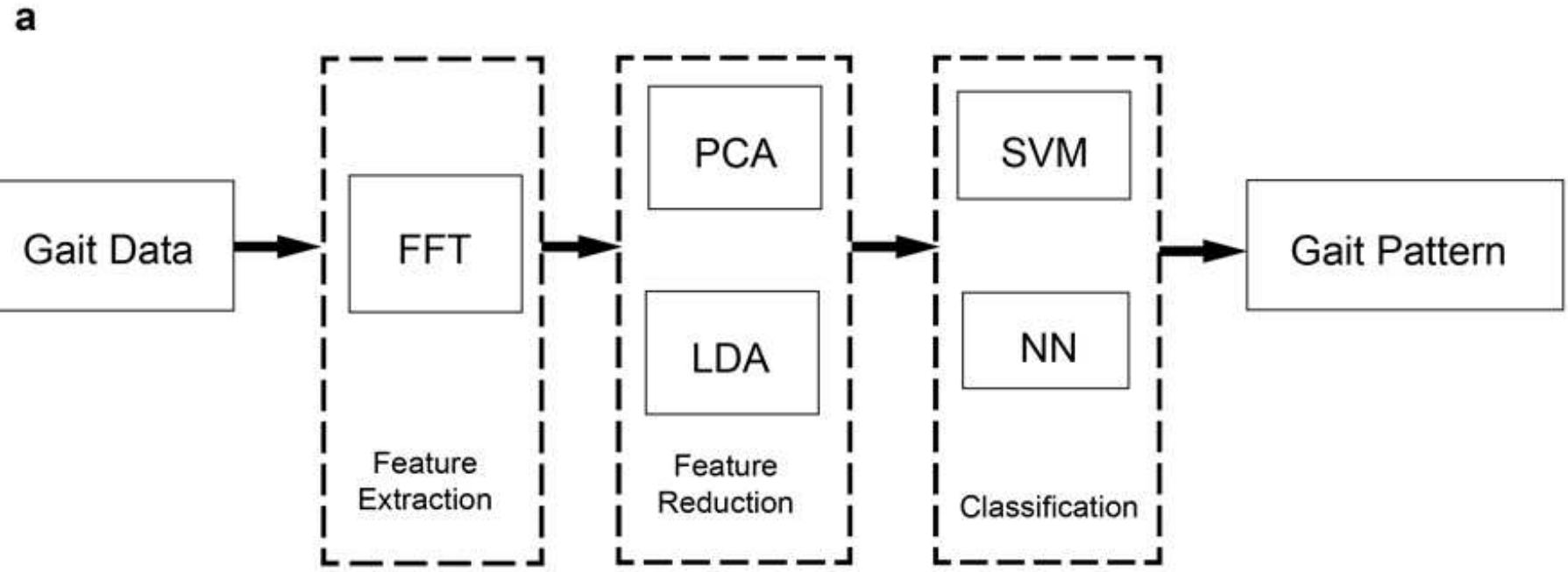

b

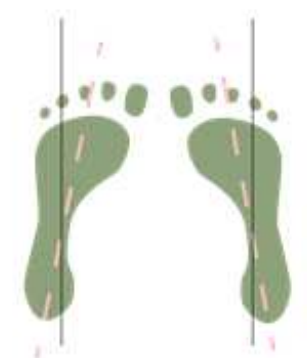

Toe in

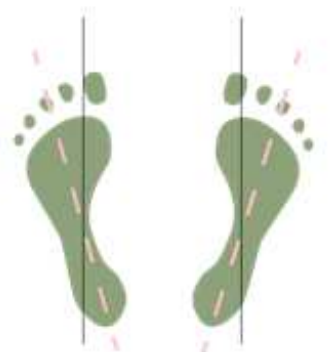

Toe out

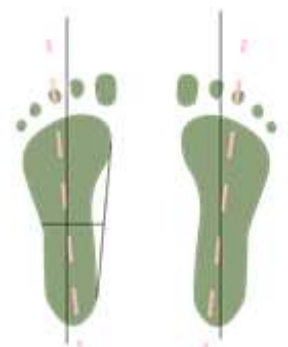

Flat

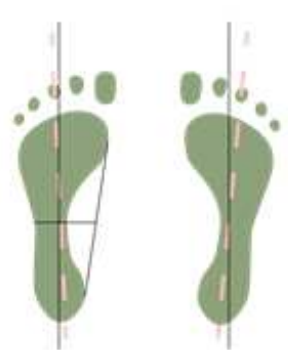

Normal 


\section{Figure 9}

a Diagram of the gait-recognition algorithm. b Gait pattern for classification - toe in, toe out, flat and normal
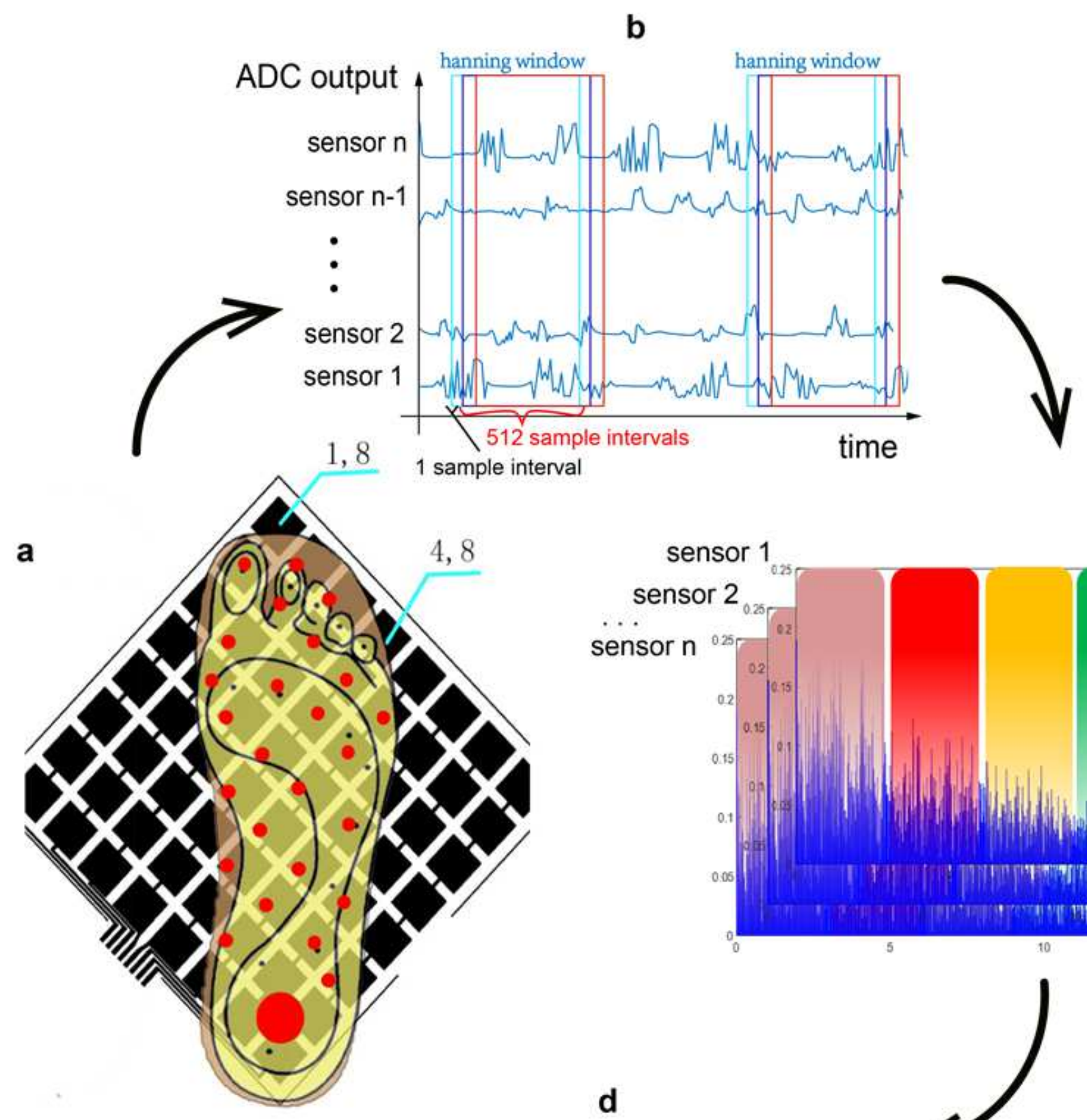

sensor 1 sensor 2 sensor $n$

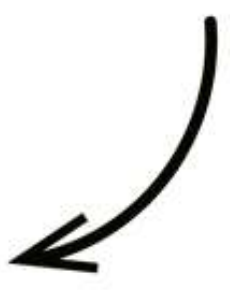

C

Figure 10

The procedure of gait feature extraction from gait cycle. a 30 sensor blocks with the red mark are selected for feature extraction. b Sliding window method (Hanning window with 512-sample intervals width) is applied to all $\mathrm{n}$ sensor blocks and transformed to frequency domain later. $\mathrm{c}$ Dividing five frequency bands 
from FFT frequency spectrum, 0 (exclude) $-2 \mathrm{~Hz}, 2$ (exclude) $-4 \mathrm{~Hz}, 4$ (exclude) $-6 \mathrm{~Hz}, 6$ (exclude) -8 $\mathrm{Hz}$, and 8 (exclude) $-10 \mathrm{~Hz}$, and summing all the frequent value in each frequency band to generate a five-elements vector for each sensor. $d$ Joining all the five-element vector from each sensor together and normalizing to a 150 -element unit vector.
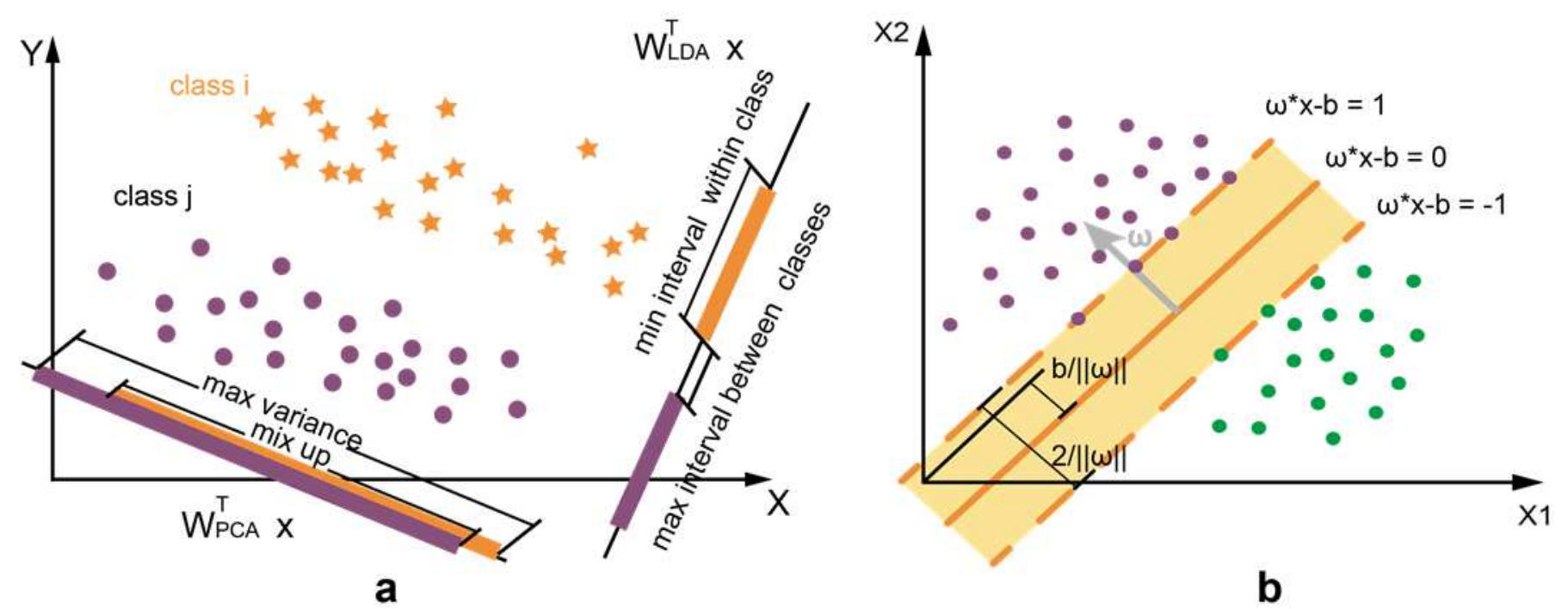

Figure 11

a LDA and PCA algorithm performance in classification in the specific situation. In this situation, LDA's performance is better than PCA's. $b$ SVM classification. The main idea of the SVM is projecting data points into a higher dimensional space, specified by a kernel function, and computing a maximummargin hyperplane decision surface that separates the two classes 